\title{
COMPENSATED COMPACTNESS AND GENERAL SYSTEMS OF CONSERVATION LAWS \\ BY
}

\author{
RONALD J. DI PERNA ${ }^{1}$
}

\begin{abstract}
We outline a general program and present some new results dealing with oscillations in weakly convergent solution sequences to systems of conservation laws. The analysis employs the Young measure and the Tartar-Murat theory of compensated compactness and deals with systems of hyperbolic and elliptic type.
\end{abstract}

1. Introduction. We are concerned with solutions to nonlinear systems of conservation laws of hyperbolic and elliptic type

$$
\sum_{j=0}^{m-1} \partial_{j} g_{j}(u)=0, \quad u: \Omega \subset R^{m} \rightarrow R^{n}, g_{j}: R^{n} \rightarrow R^{p},
$$

where $\partial_{j}=\partial / \partial y_{j}$. We shall outline a general program and present some new results dealing with oscillations in weakly convergent solution sequences to (1.1). We shall also treat approximate solutions generated by finite difference schemes and diffusive regularizing operators. For systems of both hyperbolic type and elliptic type we shall present some new results that establish strong convergence of sequences of exact and approximate solutions without using uniform bounds on the derivatives. Weak convergence is transformed into strong convergence using the Tartar-Murat theory of compensated compactness and the Young measure. In this paper we shall be concerned exclusively with questions of local convergence, i.e. convergence on compact subsets of the domain of definition $\Omega$. The analysis of initial and/or boundary layers is a separate topic.

Several preliminary remarks are in order concerning the standard notions of weak and strong convergence and their relationship to nonlinear differential equations. Many situations arise in which one is presented with a sequence of exact or approximate solutions $u^{\varepsilon}$ to (1.1) whose amplitude is bounded uniformly with respect to $\varepsilon$. In both the hyperbolic and elliptic settings the amplitude bound typically results from a maximum principle or energy argument and ensures local $L^{p}$ control of the form

$$
\int_{K}\left|u^{\varepsilon}\right|^{p} d y \leqslant \text { const., }
$$

where the constant depends on the compact subset $K$ in question. As a consequence of (1.2), one may at least assert the existence of a subsequence that converges in an

Received by the editors February 25, 1985.

1980 Mathematics Subject Classification. Primary 35L65, 76L05: Secondary 35B40. 35A40.

${ }^{1}$ Supported by NSF Grant MCS-83-01135. 
appropriate weak topology

$$
u=w-\lim u^{\varepsilon} .
$$

If $p=\infty$ the weak-star topology of $L^{\infty}$ is relevant. If $1<p<\infty$ the weak topology of $L^{p}$ is relevant. If $p=1$ the weak-star topology of the space $B M$ of bounded measures is relevant. It is well known that the unit ball of the spaces $L^{\infty}, L^{p}$ with $1<p<\infty$ and $B M$ are compact in the aforementioned topologies. Although these topologies are weak enough to ensure compactness, they are too weak to guarantee continuity of nonlinear state variables $g: R^{n} \rightarrow R^{p}$. Indeed, in general

$$
g\left(w-\lim u^{\varepsilon}\right) \neq w-\lim g\left(u^{\varepsilon}\right)
$$

if no uniform control on derivatives is assumed. The essential problem associated with this lack of continuity in the weak topology as expressed by (1.3) does not, of course, arise from the growth of the state variable $g$ at infinity but rather from oscillations in $u^{\varepsilon}$.

For technical simplicity we shall henceforth assume that all sequences under consideration are bounded in $L^{\infty}$ :

$$
\left|u^{\varepsilon}\right|_{\infty} \leqslant \text { const., }
$$

uniformly with respect to $\varepsilon$. A generalization to $L^{p}$ follows with appropriate growth restrictions on $g$. We remark that in the presence of a uniform $L^{\infty}$ bound (1.4) the basic notions of weak convergence coincide: convergence in the weak star-topology of $L^{\infty}$, convergence in the weak topology of $L^{p}, 1<p<\infty$, and convergence in the sense of distributions. In this situation we shall simply use the term weak convergence.

Weak convergence is classically contrasted with strong convergence. Weak convergence means convergence of local averages, i.e.

$$
u=w-\lim u^{\varepsilon} \quad \text { if and only if } \int_{K} u(y) d y=\lim \int_{K} u^{\varepsilon}(y) d y,
$$

for all compact $K$ in $\Omega$. Strong convergence means norm convergence, i.e.

$$
u=s-\lim u^{\varepsilon} \quad \text { if and only if } \lim \int_{K}\left|u(y)-u^{\varepsilon}(y)\right| d y=0
$$

for all compact $K$ in $\Omega$. Once again, the basic notions of strong convergence coincide if (1.4) is assumed: convergence in the strong topology of $L_{\text {loc }}^{1}$ coincides with convergence in the strong topology of $L_{\mathrm{loc}}, 1<p<\infty$. In this situation we shall simply use the term strong convergence.

A general problem in the theory of conservation laws (1.1) is the following: Given a weakly convergent sequence of exact or approximate solutions $u^{\varepsilon}$ to (1.1), determine whether or not $u^{\varepsilon}$ contains a strongly convergent subsequence. If $u^{\varepsilon}$, or a subsequence thereof, converges strongly, one may pass to the limit in a general state variable $g$ : If $g$ is continuous from $R^{n}$ to $R^{p}$ then $g$ is continuous in the strong topology, i.e.

$$
g\left(s-\lim u^{\varepsilon}\right)=s-\lim g\left(u^{\varepsilon}\right) .
$$

As a corollary one may assert that the strong limit of sequence of solutions to (1.1) is again a solution to (1.1). In contrast, one may not assert in general that the weak limit of sequence of solutions to (1.1) is again a solution to (1.1) without appealing to additional information. 
A specific problem in this area is to determine the circumstances under which strong convergence may be deduced from weak convergence without using uniform derivative estimates. We remark in passing that, if one either assumes or proves a uniform bound on derivatives in a pointwise or average sense, then weak convergence is immediately transformed into strong convergence by classical functional analysis. If $u^{\varepsilon}$ converges weakly to $u$ and if, for example, the gradients are uniformly bounded in $L$ Poc

$$
\int_{K}\left|\nabla u^{\varepsilon}\right|^{p} d y \leqslant \text { const. }
$$

then $u^{\varepsilon}$ converges strongly to $u$. In general, weak convergence plus compactness in the strong topology implies strong convergence. If a priori derivative estimates are available, no difficulty arises in proving convergence of approximation methods.

The general problem with which we are concerned may be stated as follows: Describe the oscillations in a weakly convergent sequence of functions $z^{\varepsilon}: R^{m} \rightarrow R^{N}$ subject to linear differential constraints of the form

$$
\sum_{j=0}^{m-1} A_{j} \partial_{j} z^{\varepsilon}=\varphi^{\varepsilon}
$$

and nonlinear algebraic constraints of the form

$$
\left\{z^{\varepsilon}(y)\right\} \subset M
$$

for almost all $y$ in $R^{m}$. Here $A_{j}$ denotes a constant $s \times N$ matrix with $s$ arbitrary and fixed. $M$ is a subset of the ambient state space $R^{N}$ and is usually a manifold. In the context of hyperbolic conservation laws (1.1), the distributions $\varphi^{\varepsilon}$ typically vanish or maintain a distinguished sign reflecting the presence of an entropy condition. In the context of elliptic systems the distributions $\varphi^{\varepsilon}$ typically vanish. Thus, for many purposes and, in particular, for the purpose of this introduction one may regard the object of study as a weakly convergent sequence of solutions or subsolutions to a first order system of differential equations with values in a submanifold of state space.

We remark in passing that the study of approximation methods for (1.1) involves distributions $\varphi^{\varepsilon}$ that represent singular perturbations, namely higher order discrete or analytic operators multiplied by a small coefficient $\varepsilon$. In this situation it turns out that the natural condition on the sequence $\varphi^{\varepsilon}$ is that it lies in a compact subset of the negative Sobolev space $W^{-1,2}$. The detailed structure of $\varphi^{\varepsilon}$ does not play an essential role in the compactness theory for conservation laws; $\varphi^{\varepsilon}$ is treated as a lower-order term. We refer the reader to the introductory section of [6] for a discussion of the $W^{-1,2}$ compactness condition and for applications of the compensated compactness theory to finite difference schemes.

A specific goal in this program is to describe the Young measure associated with a weakly convergent sequence $z^{\varepsilon}$ which satisfies linear differential constraints (1.5) and nonlinear algebraic constraints (1.6). We are particularly interested in determining how differential and algebraic constraints collaborate to suppress oscillations in $z^{\varepsilon}$. The problems are to be posed and answered in terms of the coefficient matrices $A_{j}$ and the constitutive set $M$. 
The general framework as presented by (1.5) and (1.6) is discussed in detail in $\$ 2$ along with the relevant background dealing with the Young measure. $\$ 2$ includes a self-contained introduction to compensated compactness and the Young measure. The prototypical examples from mechanics associated with hyperbolic, elliptic and mixed-type systems are discussed in $\S \S 2,5,7$ and 10 .

In the setting of mechanics the domain space $R^{m}$ and the range space $R^{N}$ of $z^{\varepsilon}$ represent a physical space and a state space, respectively. The differential constaints (1.5) express the basic conservation laws of mass, momentum and energy that govern general media. The algebraic constraints (1.6) embody the constitutive relations that characterize a specific medium. The equations of compressible fluid dynamics and of compressible elastostatics provide basic models for the general theory concerning hyperbolic and elliptic systems, cf. $\$ \S 5$ and 7 . The small disturbance equation of transonic flow provides the basic model for a system of mixed type; cf. $\S 10$.

One of the goals is to identify those features of the algebraic structure of the symbol of the differential system (1.5) and the constitutive manifold $M$ that admit or exclude oscillations in the sequence $z^{\varepsilon}$. In this paper we are primarily concerned with structural relationships between (1.5) and (1.6) that exclude oscillations from the sequence $z^{\varepsilon}$, in particular with mechanisms shared by hyperbolic and elliptic systems.

In the context of hyperbolic conservation laws we shall be concerned mainly with nondegenerate systems in one space dimension. A standard structural hypothesis for this class of systems is the condition of genuine nonlinearity in the sense of Lax [11], i.e. strict monotonicity of the wave speeds as a function of the wave amplitude. The prototypical examples of genuinely nonlinear systems arise in elasticity and consist of systems of two equations, $p=2$, that express the conservation laws of mass and momentum for general media, i.e. gases, liquids and solids. In the special case of solution sequences to this class of genuinely nonlinear systems of two conservation laws, we shall prove a general conjecture of Tartar [16] and establish, as a corollary, strong convergence without derivative estimates.

Tartar's conjecture may be stated roughly as follows: If the differential system (1.5) does not allow any codimension-one oscillations with values in the constitutive set $M$, then the sequence $z^{\varepsilon}$ contains no strong oscillations, i.e. $z^{*}$ converges in the strong topology. This conjecture leads to the general problem of determining the circumstances under which the removal of the highest order oscillations, namely the codimension-one oscillations, guarantees the absence of all oscillations. The evidence accumulated so far indicates that the conjecture is true at least in the case of small oscillations, i.e. in the case where

$$
\left|z^{\varepsilon}-\bar{z}\right|_{x} \ll 1
$$

for some fixed state $\bar{z}$. In general one may anticipate the necessity of formulating a hierarchy of conditions associated with certain canonical oscillations in order to eliminate all possible strong oscillations for systems in several dimensions. At the current stage of development, attention is focused on the implications of the removal of codimension-one oscillations. 
A precise formulation of Tartar's conjecture is given in $\S 2$. It turns out that a geometric condition determines whether or not it is possible for a general weakly convergent sequence $z^{\varepsilon}$ satisfying differential and algebraic constraint to contain codimension-one oscillations. The condition is stated in the state space $R^{N}$ in terms of the separation of the wave cone $\Lambda$ of (1.5) and the constitutive set $M$. It is conjectured that if $\Lambda$ and $M$ are separated, i.e. if (1.5) and (1.6) jointly exclude codimension-one oscillations, then the Young measure associated with $z^{\varepsilon}$ is a Dirac mass, i.e. $z^{\varepsilon}$ converges strongly.

In $\S \S 3,4$ and 10 we shall be concerned in part with the formulation of structural hypothesis on the flux functions $g_{j}$ of (1.1) which guarantee the separation of the associated wave cone $\Lambda$ from the constitutive manifold $M$. In this regard we first note that the separation property does not hold for hyperbolic systems in one (and several) space dimension(s):

$$
\partial_{t} u+\partial_{x} f(u)=\varphi, \quad u: R^{p} \rightarrow R^{p}
$$

cf. §3. There is no loss of generality in assuming that the flux function $g_{0}$ is the identity. We show in $\S 4$, however, that if the system (1.7) is genuinely nonlinear in the sense of Lax then the following augmented system of $p+1$ equations that includes a Lax entropy form does have the separation property:

$$
\begin{gathered}
\partial_{t} u_{i}+\partial_{x} f_{i}(u)=\varphi_{i}, \quad 1 \leqslant i \leqslant p, \\
\partial_{t} \eta(u)+\partial_{x} q(u)=\varphi_{p+1} .
\end{gathered}
$$

Here $(\eta, q)$ denotes any generalized entropy pair for (1.7) for which $\eta$ is strictly convex. The separation property is an immediate corollary of a result of Lax [12] which implies that there exist no shock waves in a genuinely-nonlinear system that conserves a strictly convex entropy field. For example, in the setting of genuinely nonlinear elasticity there exist no shock waves that simultaneously conserve mass, momentum and mechanical energy.

It is appropriate to remark at this point that, for hyperbolic systems of conservation laws with degenerate wave speeds, it is not possible in general to separate with wave cone from the constitutive manifold by augmenting the system with a finite number of entropy forms. In particular, if one or more of the wave speeds is linearly degenerate and thus allows contact discontinuities, then codimension-one oscillations may exist. The intersection of the wave cone and the manifold has a relatively simple structure and leads to an interesting problem of determining the nontrivial structure of the Young measure.

In $\S \S 5$ and 9 we consider a class of systems of two equations (1.7) with the form of the Lagrangian equations of elasticity, i.e.

$$
\partial_{t} v-\partial_{x} u=\varphi_{1}, \quad \partial_{t} u-\partial_{x} \sigma(v)=\varphi_{2} .
$$

We recall that (1.8) is strictly hyperbolic if $\sigma^{\prime}>0$ and genuinely nonlinear if $\sigma^{\prime \prime} \neq 0$. We introduce a doubly augmented system of four equations involving two natural entropy forms,

$$
\begin{aligned}
\partial_{t} v-\partial_{x} u & =\varphi_{1}, \quad \partial_{t} u-\partial_{x} \sigma=\varphi_{2}, \\
\partial_{t} \eta_{j}+\partial_{x} q_{j} & =\varphi_{j+2} \quad(j=1,2),
\end{aligned}
$$


and we prove that the Young measure associated with any weakly convergent sequence $\left(u^{\varepsilon}, v^{\varepsilon}\right)$ having uniformly small oscillation is a Dirac mass if $\sigma^{\prime}>0$ and $\sigma^{\prime \prime} \neq 0$; cf. Theorem 5.1. As stated above, each of the sequences of distributions $\varphi_{j}\left(u^{\varepsilon}, v^{\varepsilon}\right), 1 \leqslant j \leqslant 4$, is assumed to lie in a compact subset of $W^{-1,2}$. Thus, one may show that the Young measure reduces to a Dirac mass, i.e. a monatomic measure with unit mass, if its support is sufficiently small, and therefore one may show that $\left(u^{\varepsilon}, v^{\varepsilon}\right)$ converges strongly. The reduction of the Young measure $\nu$ is established with the aid of a basic functional equation for $\nu$ introduced by Tartar [15] which will be discussed below.

We remark that the entropy forms in the augmented system (1.9) arise naturally from Noether's theorem through the temporal and spatial translation invariance of the integrand in the associated variational principle; cf. $\$ 5$. The pair of state variables $\left(\eta_{1}, q_{1}\right)$ denotes a generalized entropy pair associated with temporal translation invariance: $\eta_{1}$ is the mechanical energy and $q_{1}$ is the negative power supplied by the stress tensor,

$$
\eta_{1}=\frac{1}{2} u^{2}+\Sigma(v), \quad q_{1}=-u \sigma(v),
$$

where the stored-energy function $\Sigma$ is the primitive of $\sigma$. The dual entropy pair $\left(\eta_{2}, q_{2}\right)$ is associated with spatial translation invariance and is given by

$$
\eta_{2}=u v, \quad q_{2}=\frac{1}{2} u^{2}+\tau(v),
$$

where $\tau$ is the Legendre transform of $\Sigma$. The duality is a reflection of the fact that the Lagrangian equations are invariant under the simultaneous interchange of space with time and stress with strain.

As a corollary of the aforementioned compactness Theorem 5.1 for elasticity, we may state the following result: Suppose $\left(u^{\varepsilon}, v^{\varepsilon}\right)$ is a weakly convergent sequence of solutions to the system

$$
\partial_{t} v-\partial_{x} u=0, \quad \partial_{t} u-\partial_{x} \sigma(v)=0 .
$$

If $\left(u^{\varepsilon}, v^{\varepsilon}\right)$ has uniformly small oscillation and if each of the following sequences of entropy fields

$$
\partial_{t} \eta_{j}\left(u^{\varepsilon}, v^{\varepsilon}\right)+\partial_{x} q_{j}\left(u^{\varepsilon}, v^{\varepsilon}\right), \quad j=1,2,
$$

given by (1.10) and (1.11) lies in a compact subset of $W^{-1.2}$, then $\left(u^{\varepsilon}, v^{\varepsilon}\right)$ converges strongly. In this connection we note that if the solutions $\left(u^{\varepsilon}, v^{\varepsilon}\right)$ satisfy the Lax entropy condition, then one may easily verify the compactness of the entropy fields using just the fact that the $L^{\infty}$ norm of $\left(u^{\varepsilon}, v^{\varepsilon}\right)$ is uniformly bounded. Thus, the hypothesis of compact entropy fields is natural from the viewpoint of both initial and boundary value problems.

We mention this corollary in connection with previous work on strong convergence of solutions to hyperbolic conservation laws using compensated compactness $[6,7]$. In [6] it was shown that, for a general class of systems of two genuinely nonlinear conservation laws in one space dimension, the $W^{-1,2}$-compactness of all entropy fields implies the $L^{1}$-strong compactness of solutions. This result applies, in particular, to solutions that satisfy the Lax entropy inequality: If $u^{\varepsilon}$ is a uniformly 
bounded sequence of solutions satisfying the Lax entropy inequality [12] then all entropy fields are compact. This work leads to the question of whether or not knowledge of the compactness of just the physical entropy fields is sufficient to deduce the compactness of uniformly bounded solutions. The question can be answered in the affirmative at least in the setting of Lagrangian elasticity and small oscillations.

The compactness results of $[6,7]$ for hyperbolic systems of two conservation laws were also established by showing that the Young measure $\nu$ associated with a weakly convergent solution sequence reduces to a Dirac mass. The proof of reduction of the Young measure $\nu$ is based on an analysis of a commutativity relation of the form

$$
\nu \circ B=B \circ \nu
$$

where $B$ denotes the symplectic (antisymmetric, bilinear) form acting on all entropy pairs. Equation (1.13) is shorthand for the statement that

$$
\langle\nu, \eta \tilde{q}-\tilde{\eta} q\rangle=\langle\nu, \eta\rangle\langle\nu, \tilde{q}\rangle-\langle\nu, \tilde{\eta}\rangle\langle\nu, q\rangle
$$

for all entropy pairs $(\eta, q)$ and $(\tilde{\eta}, \tilde{q})$. The brackets in (1.14) denote the expected value of the indicated variable, e.g.

$$
\langle\nu, \eta\rangle=\int_{R^{p}} \eta(\lambda) d \nu(\lambda) .
$$

The functional equation (1.14) was introduced by Tartar in [15]; if all entropy fields are compact, then the Young measure $\nu$ commutes with $B$ acting on all entropy pairs. In [15], Tartar conjectured that any probability measure satisfying (1.14) for all entropy pairs of a nondegenerate hyperbolic system (1.7) is a Dirac mass and proved the conjecture in the case of a scalar equation. The conjecture was subsequently verified for nondegenerate systems of two equations using the Lax progressing entropy waves [6, 7]. As a corollary, the first large data existence theorems for the Cauchy problem for elasticity and isentropic gas dynamics were obtained along with the first convergence results for first order accurate finite difference schemes; cf. $[6,7]$. We refer the reader to the introductory sections of [6] for additional background and a more precise formulation of results dealing with Tartar's functional equation (1.14) in setting of nondegenerate systems. More recently, the functional equation (1.14) has been analyzed for degenerate systems of two hyperbolic conservation laws by D. Serre [19].

In $\$ 10$, we show that the wave cone and constitutive manifold are separated for the small disturbance equations of transonic flow augmented by a single convex entropy form that is associated with spatial translation invariance in either of the two independent variables. The small disturbance equations provide the canonical example of a system of two conservation laws which changes type from hyperbolic to elliptic as the state variable crosses a hypersurface. Thus, the separation hypothesis of Tartar's conjecture is relevant.

$\S \S 6$ and 7 are concerned with the compactness of solutions to general elliptic systems in two independent variables and to the special equations of hyperelasticity having a stored-energy function that is polyconvex in the sense of J. Ball [2]. In §6, 
we establish the strong compactness of solutions with small oscillation to general elliptic systems (1.1) without using derivative estimates. It is shown that if the support of the associated Young measure is sufficiently small then it must reduce to a Dirac mass. Unlike the hyperbolic case, additional nonlinear entropy forms are not required to establish compactness for elliptic solution sequences, at least locally. We also obtain a similar result dealing with compactness of solutions with large oscillation for a special class of elliptic systems. The proofs are based upon a study of the coercive algebraic structure of the equations in state space and employ the basic div-curl lemma of Tartar and Murat.

In $\S 7$ we consider the equations of hyperelasticity with a strictly polyconvex stored energy function and establish compactness of solution sequences with small oscillation: If $u^{\varepsilon}$ is a sequence of hyperelastic deformations with small oscillation, then there exists a subsequence which converges in the strong topology of $L_{\text {loc }}^{1}$. The proof is based upon a decomposition of the basic elliptic form associated with the system into a canonical quadratic part and a null-Lagrangian form. We refer the reader to J. Ball $[2,3]$ for a general discussion of elasticity in the context of the calculus of variations and for an introduction to the notions of polyconvexity and nullLagrangian forms.

In the setting of elliptic systems (1.1) in two independent variables, our proof of compactness of solution sequences with small oscillation is based on a study of Tartar's functional equation (1.14). In the context of elliptic equations the flux functions $\left(g_{i 0}, g_{i 1}\right)$ act as "entropy pairs". If the Young measure commutes with the bilinear form $B$ acting on just the flux functions of an elliptic system, then it must reduce to a point mass if its support is sufficiently small. Thus, the commutativity relation (1.14) provides a viewpoint for studying compactness to systems of both hyperbolic and elliptic type in two independent variables. One topic for future investigation deals with the structure of the Young measure for systems of mixed type in two independent variables.

These results on 2-d elliptic systems and on polyconvex elasticity provided motivation for a general theorem of Tartar [16] that establishes compactness of solution sequences with small oscillation to elliptic systems in an arbitrary number of state variables. This theorem follows as a corollary of a general result [16] that guarantees compactness provided that the wave cone is separated from the tangent space to the manifold. In other words, if the wave cone is separated from the constitutive manifold by a hyperplane, then the Young measure reduces to a point mass if its support is sufficiently small.

It turns out that this type of maximal separation of wave cone and manifold occurs if and only if the system is elliptic. In contrast, if the system is hyperbolic, then the wave cone intersects the tangent plane to the constitutive manifold in a maximal number of directions, although it may miss the manifold itself if appropriate entropy forms are introduced, cf. \$3. These observations motivate the general problem of determining the geometric relationship between the wave cone and constitutive manifold for general systems of conservation laws in the context of the Young measure. The augmented system (1.9) of Lagrangian elasticity exhibits, 
roughly speaking, a fourth order separation between wave cone and manifold which can be utilized to prove that the Young measure is a Dirac mass with the aid of the basic div-curl lemma of Tartar and Murat from the compensated compactness theory. This mild form of separation provides an interesting contrast with the second order separation presented by elliptic systems.

The program discussed here deals with the structure of oscillations at a fixed point in space and time. We refer the reader to [8 and 17] for work on the dynamic behavior.

2. General structure. We shall begin with a discussion of general systems and then turn to mechanics. Consider a sequence of measurable maps $z^{\varepsilon}: R^{m} \rightarrow R^{N}$ subject to the following three conditions. First, the sequence $z^{\varepsilon}$ converges in the weak-star topology of $L^{\infty}$ :

$$
z=w-\lim z^{\varepsilon} .
$$

Second, $z^{\varepsilon}$ solves a linear constant coefficient system of differential equations

$$
\sum_{j=0}^{m-1} A_{j} \partial_{j} z^{\varepsilon}=0,
$$

where $A_{j}$ is an $s \times N$ matrix. Third, the values of $z^{\varepsilon}$ lie in a fixed subset of $R^{N}$ for almost all $y$ in $R^{m}$ :

$$
z^{\varepsilon}(y) \in M \subset R^{N} .
$$

Problem. Describe the Young measure associated with the sequence $z^{\varepsilon}$.

In the setting of mechanics $z^{\varepsilon}$ maps a physical domain to a state space, or some proper subsets thereof. The set $M$ is typically a low dimensional manifold which expresses the constitutive laws of the system. A general system of $p$ conservation laws in $n$ unknowns,

$$
\sum_{j=0}^{m-1} \partial_{j} g_{j}(u)=0, \quad g_{j}: R^{n} \rightarrow R^{p},
$$

fits into the general framework as follows: The state space $R^{N}$ has dimension $N=p m$ : one component $w_{i j}$ is associated with each of the flux functions $g_{i j}$ in (2.4), $1 \leqslant i \leqslant p, 1 \leqslant j \leqslant m$. The differential constraints (2.2) assert that the divergence of each row of the matrix $w_{i j}$ vanishes, i.e.

$$
\sum_{j=0}^{m-1} \partial_{j} w_{i j}=0, \quad 1 \leqslant i \leqslant p .
$$

The algebraic constraints (2.3) are expressed by an $n$-dimensional set defined in terms of the flux functions $g_{i j}$ :

$$
M=\left\{w \in R^{N}: w_{i j}=g_{i j}(u) \text { for some } u \text { in } R^{n}\right\} .
$$

Thus, the system (2.4) is viewed as linear in $g$ and nonlinear in $u$. At this point, the type of the system is unspecified. We shall see below that the type is determined by the tangent plane to the constitutive set $M$. 
More generally, we are interested in describing the oscillations in weakly convergent sequences $z^{\varepsilon}$ to inhomogeneous systems of the form

$$
\sum_{j=0}^{m-1} A_{j} \partial_{j} z^{\varepsilon}=\varphi^{\varepsilon},
$$

where the sequence of distributions $\varphi^{\varepsilon}$ is contained in a compact subset of $W_{\operatorname{loc}}^{-1.2}\left(R^{m}\right)$. For example, the method of artificial viscosity applied to the Cauchy problem for Lagrangian elasticity (1.8) leads to the problem of analyzing the convergence of solutions $\left(u^{\varepsilon}, v^{\varepsilon}\right)$ to the parabolic system

$$
\partial_{t} v^{\varepsilon}-\partial_{x} u^{\varepsilon}=\varepsilon \partial_{x}^{2} v^{\varepsilon}, \quad \partial_{t} u^{\varepsilon}-\partial_{x} \sigma\left(v^{\varepsilon}\right)=\varepsilon \partial_{x}^{2} u^{\varepsilon}
$$

as the viscosity parameter $\varepsilon$ vanishes. Here the components of $\varphi^{\varepsilon}$ are given by second order operators. We refer the reader to [6] for convergence results dealing with (2.8).

The Young MEASURE. One of the goals is to determine restrictions on the Young measure associated with a weakly convergent sequence subject to differential and algebraic constraints. Several preliminary remarks are in order. Consider an arbitrary weakly convergent sequence of functions $z^{\varepsilon}$ and let $h$ denote an arbitrary continuous real-valued map on the state space $R^{N}$. The Young measure provides a representation of composite weak limits as follows; cf. [15].

THEOREM 2.1. There exists a subsequence of $z^{\varepsilon}$ (which will not be relabeled) such that for all $h$ the weak limit of the composition exists:

$$
\bar{h}=w-\lim h\left(z^{\varepsilon}\right) .
$$

Second there exists a parametrized family of nonnegative Radon measures $\nu_{y}$ on $R^{N}$ with unit mass such that the composite limit $\bar{h}$ coincides almost everywhere with the expected value of $h$ :

$$
\bar{h}(y)=\left\langle\nu_{y}, h\right\rangle \equiv \int_{R^{N}} h(\lambda) d \nu_{y}(\lambda) .
$$

REMARK 1. We emphasize that one family of probability measures $\left\{\boldsymbol{\nu}_{y}\right\}$ suffices to simultaneously describe all composite limits, after passage to a subsequence. Of course, different Young measures $\nu$ may be associated with different subsequences.

REMARK 2. Formally, the weight which $\nu_{y}$ assigns to a subset $E$ of $R^{N}$ equals the probability that the vectors $z^{\varepsilon}(y)$ lie in $E$ in the limit

$$
\nu_{y}(E)=\lim \operatorname{prob}\left\{z^{\varepsilon}(y) \in E\right\}
$$

Following standard practice, we shall refer to a nonnegative measure with unit mass as a probability measure, independently of the presence or absence of a probabilistic interpretation.

A useful characterization of strong convergence is the following:

COROLlaRY 2.1. The sequence $z^{\varepsilon}$ converges strongly to $z$ if and only if the Young measure is a Dirac mass: $\nu_{y}=\delta_{z(y)}$, for almost all $y$.

We recall that strong convergence guarantees the continuity of arbitrary state variables, i.e. the commutator

$$
C \equiv w-\lim h\left(z^{\varepsilon}\right)-h\left(w-\lim z^{\varepsilon}\right)
$$


vanishes for all $h$. There are many circumstances in which sustained oscillations prevent strong convergence and produce a nontrivial commutator $C$. In general, the deviation between weak and strong convergence is captured by the commutator $C$ and is expressed by the manner in which the representing measure $\nu$ is distributed over state space. One basic example of non-Dirac structure is provided by a sequence formed from the scaling of a periodic function $v$ of one variable of period $p$

$$
z^{\varepsilon}(y)=v(y \cdot \xi / \varepsilon)
$$

where $\xi$ is fixed. It is easy to show that $z^{\varepsilon}$ converges weakly to a constant function whose value equals the normalized area of the graph of the structure function $v$ :

$$
w-\lim z^{\varepsilon}=\frac{1}{p} \int_{0}^{p} v(t) d t
$$

The act of composing with $h$ also yields a scaled periodic function

$$
h\left(z^{\varepsilon}(y)\right)=\{h \circ v\}(y \cdot \xi / \varepsilon) .
$$

For the same reason,

$$
w-\lim h\left(z^{\varepsilon}\right)=\frac{1}{p} \int_{0}^{p} h(v(t)) d t
$$

The right-hand side of (2.9) is clearly a bounded linear functional acting on the space of continuous state variables and is therefore a measure. Here, the Young measure is independent of $y$ and is given by the image of normalized Lebesgue measure under the structure function $v$, i.e.

$$
\nu_{y}=v \frac{d t}{p}, \quad\left\langle\nu_{y}, h\right\rangle=\frac{1}{p} \int_{0}^{p} h(v(t)) d t
$$

The Classification OF WEAKLY CONTINUOUS STATE VARIABLES. The first step of the program is to determine the restrictions on $\nu$ that follow from the differential constraint (2.7), without taking $M$ into account. To this end, one of the goals is to classify the state variables which are weakly continuous when restricted to approximate solution sequences $z^{\varepsilon}$. In other words, the first step is to describe the maps $h$ which commute with $\nu$ when $\varphi^{\varepsilon}$ lies in a compact subset of $W^{-1,2}$; the commutator $C$ vanishes if and only if

$$
h\left(\left\langle\nu_{y}, \lambda\right\rangle\right)=\left\langle\nu_{y}, h(\lambda)\right\rangle
$$

for almost all $y$.

The classification of state variables which satisfy $(2.10)$ is one of the subjects addressed by the theory of compensated compactness. The results which are currently available on the classification problem and which we shall discuss below are formulated in terms of a variety $V$ associated with the symbol of the differential system (2.2). The variety $V$ was introduced by Tartar and Murat.

A related problem is to classify the weakly lower continuous state variables, i.e. the functions $h$ which satisfy

$$
h\left(w-\lim z^{\varepsilon}\right) \leqslant w-\lim h\left(z^{\varepsilon}\right)
$$


or equivalently

$$
h\left(\left\langle\nu_{y}, \lambda\right\rangle\right) \leqslant\left\langle\nu_{y}, h(\lambda)\right\rangle
$$

if $\varphi^{\varepsilon}$ lies in a compact subset of $W^{-1,2}$. The information contained in (2.10) and (2.11) constitutes one of the principle restrictions on the Young measure $\nu$ which in combination with $M$ leads to a reduction of $\nu$ to a Dirac mass in certain hyperbolic and elliptic circumstances to be discussed below.

REMARK. If $z^{\varepsilon}$ satisfies the uniform $L^{\infty}$ bound (2.7) then the support of $\nu_{y}$ is contained in a ball of radius $r$ and all expected values are well defined.

THE OSCILlation VARIETY $V . V$ is a variety in the product of physical space and state space $R^{m} \times R^{N}$ consisting of pairs $(\xi, \lambda)$ such that $\lambda$ lies in the nullspace of the symbol of (2.2) with normal $\xi$ :

$$
V=\left\{(\xi, \lambda): \sum_{j=0}^{m-1} \xi_{j} A_{j} \lambda=0, \xi \neq 0\right\} .
$$

Thus, the projection of $V$ onto physical space $R^{m}$ is the standard characteristic cone $V_{c}$ of nonelliptic directions of (2.2) in physical space. The projection of $V$ onto state space $R^{N}$ yields a cone $\Lambda$, which we shall refer to as the wave cone, consisting of directions $\lambda$ that may be regarded as the nonelliptic directions of state space

$$
V_{c} \equiv P_{m} V, \quad \Lambda=P_{N} V \text {. }
$$

In general, for each nonelliptic direction $\xi$ of physical space, there exist many nonelliptic directions $\lambda$ of state space and conversely.

The relevance of $V$ for the study of oscillations stems from the fact that each point of $V$ corresponds to a canonical codimension-one oscillating solution sequence of (2.2). With this sequence one can test a given state variable for the virtue of weak continuity $(2.10)$ or weakly lower continuity $(2.11)$, i.e. derive necessary conditions for the Young measure to satisfy (2.10) or (2.11).

The canonical sequence is constructed as follows. A point $(\xi, \lambda)$ lies in $V$ if and only if (2.2) admits a solution consisting of two constant states that are separated in physical space by a hyperplane with normal $\xi$ and that differ in state space by a wave vector $\lambda$ :

$$
z=z(y ; a, b, \xi)=\left\{\begin{array}{ll}
a & \text { if }(y, \xi)>0 \\
b & \text { if }(y, \xi)<0
\end{array}\right\},
$$

where $a-b=\lambda \in \Lambda$. The defining equation for $V$ is merely the Rankine-Hugoniot relation for a jump discontinuity from state $a$ to state $b$ across a hyperplane with normal $\xi$ : The characteristic hyperplanes of (2.2) are those whose normal lics in the characteristic cone $P_{m} V$.

The relationship of $V$ codimension-one oscillations is due to the translation and scale invariance of (2.2). Each point $(\xi, \lambda)$ of $V$ corresponds to a piecewise constant periodic solution $z_{p}$ which is constant on hyperplanes and which oscillates between $a$ and $b$ at any prescribed frequency $\theta$, namely

$$
\begin{gathered}
z=z_{p}(y ; a, b, \xi, \theta) \\
z_{p}=\left\{\begin{array}{ll}
a & \text { if } n \theta<(y, \xi)<(n+1) \theta, n \text { even } \\
b & \text { if } n \theta<(y, \xi)<(n+1) \theta, n \text { odd }
\end{array}\right\} .
\end{gathered}
$$


As a consequence of the scale invariance of (2.2) each point of $V$ corresponds to an elementary oscillating sequence in the form of a scaled periodic function

$$
z^{\varepsilon}=z_{p}(y / \varepsilon ; a, b, \xi, \theta)
$$

that converges weakly to a constant function whose value is the vector $\theta a+(1-\theta) b$ on the line segment between $a$ and $b$ determined by the frequency $\theta$ : the Young measure for $z^{\varepsilon}$ is diatomic

$$
\nu_{y}=\nu=\theta \delta_{a}+(1-\theta) \delta_{b} .
$$

Using this special test sequence $z^{\varepsilon}$ one may establish a simple necessary condition for weak continuity and weak lower continuity. These conditions turn out to be sufficient conditions for quadratic maps. Hence for quadratic maps weak continuity and weak lower continuity with respect to codimension-one solution sequences imply weak continuity and weak lower continuity for all approximate solution sequences. The following basic theorem is due to Tartar and Murat [16]:

THEOREM 2.2. The map $Q(\lambda)=\sum b_{i j} \lambda_{i} \lambda_{j}$ is weakly continuous when restricted to function sequences for which the right-hand side $\varphi^{\varepsilon}$ of (2.2) lies in a compact subset of $W_{\mathrm{loc}}^{-1.2}$ if and only if

$$
\left(d^{2} / d t^{2}\right) Q(a+t \lambda)=0, \quad \text { for all } a \in R^{N}, \lambda \in \Lambda .
$$

The map $Q$ is weakly lower continuous if and only if

$$
\left(d^{2} / d t^{2}\right) Q(a+t \lambda) \geqslant 0, \text { for all } a \in R^{N}, \lambda \in \Lambda \text {. }
$$

REMARK 1. Conditions (2.14) and (2.15) state respectively that $Q$ is affine and convex along the rays determined by the wave cone $\Lambda$. If no differential constraints are present, i.e. if all $A_{j}$ vanish, then $\Lambda=R^{N}$, and we revert to the classical situation where, in general, the only weakly continuous maps are affine and the only weakly lower continuous maps are convex.

RemarK 2. Since $Q$ is quadratic, (2.14) means that the bilinear form $B=\left(b_{i j}\right)$ vanishes on the wave cone, i.e. $(B \lambda, \lambda)=0$ if $\lambda \in \Lambda$.

Proof of NeCESSITY. Suppose $Q$ is weakly continuous. Then

$$
w-\lim Q\left(z^{\varepsilon}\right)=Q\left(w-\lim z^{\varepsilon}\right)=Q(\theta a+(1-\theta) b) .
$$

We observe that the composition $Q\left(z^{\varepsilon}\right)$ of $Q$ with the canonical sequence $z^{\varepsilon}$ is a piecewise constant function that oscillates between $Q(a)$ and $Q(b)$ at the frequency $\theta$. Thus

$$
w-\lim Q\left(z^{\varepsilon}\right)=\theta Q(a)+(1-\theta) Q(b) .
$$

We conclude that $Q$ is affine on line segments whose end points differ by a wave vector, i.e.

$$
\theta Q(a)+(1-\theta) Q(b)=Q(\theta a+(1-\theta) b)
$$

if $a-b=\lambda$. The convexity statement (2.15) is treated similarly.

For the purposes of this paper we shall make use of just quadratic maps $Q$ satisfying (2.14) and (2.15). With regard to classification results for general polynomial maps we refer the reader to the work of F. Murat [14] dealing with systems with a constant rank symbol. 
One may anticipate that information from the full variety $V$ will be required, among other things, to resolve general questions on oscillations. However, at the present stage of development, we shall be concerned with the information that can be obtained from the study of its projection $\Lambda$ onto state space. To this end, the main tool is provided by the compensated compactness Theorem 2.2 , which yields a Jensen-type inequality

$$
Q\left(\left\langle\nu_{y}, \lambda\right\rangle\right) \leqslant\left\langle\nu_{y}, Q(\lambda)\right\rangle
$$

for the Young measure $\nu_{y}$ associated with a weakly convergence sequence of approximate solutions $z^{\varepsilon}$ to (2.2) provided that $Q$ is quadratic and convex on the wave cone $\Lambda$. We remark in passing that if $Q$ is convex (in all directions), then (2.16) is the classical Jensen inequality and asserts the lower continuity of arbitrary convex maps.

The next step in the program is to determine the influence of the algebraic constraints (2.3) on the Young measure. Tartar's conjecture [17] in this regard states that if the differential constraints (2.2) admit no codimension-one oscillations with values in the constitutive set $M$, then the Young measure associated with $z^{\varepsilon}$ is a Dirac mass. A more precise statement may be given as follows: For each point $a$ in $R^{N}$ let $\Lambda_{a}$ denote the wave cone translated by $a$, i.e.

$$
\Lambda_{a}=a+\Lambda=\{a+\lambda: \lambda \in \Lambda\} .
$$

CONJECTURE 2.1. If the translated wave cone is separated from $M$ in the sense that

$$
\Lambda_{a} \cap M=\{a\}
$$

for all $a$, then $\nu_{y}$ is a Dirac mass for almost all $y$.

If (2.17) holds, then there do not exist canonical oscillating sequences (2.13) with values in $M$. If the Young measure can not be diatomic in the presence of (2.7) and (2.3), then it is conjectured that it must be monatomic.

In $\S \S 3,4$ and 10 , we shall be concerned with the circumstances under which the separation property (2.17) holds. In the setting of Lagrangian elasticity we shall present a proof of Conjecture 2.1 in $\$ 9$.

3. The separation hypothesis for elliptic and hyperbolic systems. In this section we shall be concerned with structural hypotheses that guarantee the separation of wave cone and constitutive manifold in the sense of (2.17). We shall consider quasilinear systems of conservation laws (2.4) consisting of $p$ equations in $n$ unknowns. System (2.4) is regarded as linear with respect to the flux functions $g$ and nonlinear with respect to the primitive variable $u$ in the sense of the decomposition (2.5)-(2.6).

LEMMA 3.1. The system (2.4) is elliptic if and only if $p=n$ and the tangent space $T_{a} M$ to $M$ at $a$ is separated from the translated wave cone $\Lambda_{a}$ in the sense that

$$
\Lambda_{a} \cap T_{a} M=a
$$

for all $a$. Here $T_{a} M$ denotes the affine space consisting of vectors of the form $a+t$, where $t$ is a tangent vector to $M$ at $a$.

LEMMA 3.2. If the system (2.4) is hyperbolic then for each point a the tangent space $T_{a} M$ intersects $\Lambda_{a}$ in a maximal number $n$ of independent directions, $n=\operatorname{dim} T_{a} M$. 
It follows from Lemma 3.1 that the separation property (2.17) holds for elliptic systems at least locally. In contrast, the separation property fails for hyperbolic systems: $\Lambda_{a}$ and $M$ have a nontrivial intersection. In the absence of an entropy condition, hyperbolic systems admit codimension-one oscillations built from parallel families of discontinuities. The role of entropy with regard to separation will be discussed in $\$ 4$.

Proof of Lemmas 3.1 AND 3.2. We recall that (2.4) is elliptic if the linearized system

$$
\sum_{j=0}^{m-1} \nabla g_{j}(\bar{u}) \partial_{j} v=0
$$

admits no real characteristic hyperplanes for all $\bar{u}$, i.e. if the symbol

$$
S(\xi)=\sum \xi_{j} \nabla g_{j}(\bar{u})
$$

associated with the normal $\xi$ is invertible for all nonzero $\xi$. Using the summation convention, we shall write the components of $S$ in the form

$$
S_{i k}(\xi)=\xi_{j} \frac{\partial}{\partial u_{k}} g_{i j}(\bar{u})
$$

in terms of the components $g_{i j}$ of $g_{j}$. We recall that by definition the wave cone $\Lambda$ consists of vectors $\lambda$ in $R^{N}$ that lie in the nullspace of the symbol $\sum \xi_{j} A_{j}$ of (2.2) for some nonzero $\xi$. Here, $\Lambda$ is contained in $R^{m p}$ and consists of matrices $\lambda_{i j}$ such that

$$
\sum_{j=0}^{m-1} \xi_{j} \lambda_{i j}=0, \quad 1 \leqslant i \leqslant p,
$$

for some nonzero $\xi$. Thus $\Lambda$ consists of singular $p \times m$ matrices.

We observe that a typical point in $\Lambda_{a}$ takes the form $b=a+\lambda$ where $\lambda$ is singular, while a typical point in $T_{a} M$ takes the form

$$
b=a+\sum_{k=0}^{n} \frac{\partial}{\partial u_{k}} g_{i j}(\bar{u}) \tau_{k}, \quad \text { where } a=\left\{g_{i j}(\bar{u})\right\},
$$

and the vector $\tau$ determines a linear combination of tangent vectors to $M$. Hence, $b$ lies in the intersection of $\Lambda_{a}$ and $T_{a} M$ if and only if the matrix $\left(\partial / \partial u_{k}\right) g_{i j}(\bar{u}) \tau_{k}$ is singular, i.e. if and only if there exists a nonzero $\xi$ such that the form

$$
S_{i k}(\xi) \tau_{k} \equiv \xi_{j} \frac{\partial}{\partial u_{k}} g_{i j}(\bar{u}) \tau_{k}
$$

vanishes for all $i, 1 \leqslant i \leqslant p$. If (2.4) is elliptic then $S(\xi)$ is invertible, $\tau$ vanishes, $b$ equals $a$, and the separation property (3.1) holds.

In order to discuss the hyperbolic case we shall distinguish a time-like variable and assume without loss of generality that $g_{0}$ is the identity map, in which case (2.4) takes the form

$$
\partial_{t} u+\sum_{j=1}^{m-1} \partial_{j} g_{j}(u)=0
$$


We shall refer to (3.3) as hyperbolic if for every $\bar{u}$ the linearized system

$$
\partial_{t} v+\sum_{j=1}^{m-1} \nabla g_{j}(\bar{u}) \partial_{j} v=0
$$

admits a maximal number of independent plane wave solutions propagating in each direction $\omega$ of space:

$$
v^{\prime}(y, t)=\varphi\left(y \cdot \omega-\mu^{\prime} t\right) r^{\prime}, \quad 1 \leqslant l \leqslant n .
$$

Here $\varphi$ is arbitrary and

$$
\left\{r^{\prime}=r^{\prime}(\omega): 1 \leqslant l \leqslant n\right\}
$$

represents a collection of $n$ independent eigenvectors associated with the $p \times n$ eigenvalue problem

$$
\left\{\mu I-\sum \omega_{j} \nabla g_{j}(\bar{u})\right\} r=0 .
$$

In short, (3.3) is hyperbolic if the matrix

$$
\sum_{j=1}^{m-1} \omega_{j} \nabla g_{j}(\bar{u})
$$

possesses $n$ independent eigenvectors for every $\omega$ in $R^{m-1}$ and every $\bar{u}$ in $R^{n}$, equivalently, if the form

$$
\sum_{j=0}^{m-1} \xi_{j}^{\prime}(\omega) \frac{\partial}{\partial u_{k}} g_{i j}(\bar{u}) r_{k}^{l}(\omega)
$$

vanishes for each $l, 1 \leqslant l \leqslant n$, and all $\omega$ where $\xi^{\prime}$ is the space-time vector

$$
\xi^{\prime}=\left\{\mu^{\prime}(\omega), \omega\right\} \in R \times R^{m-1} .
$$

Thus, for each spatial direction $\omega$, the intersection of $\Lambda_{a}$ and $T_{a} M$ contains $n$ independent matrices of the form

$$
B^{\prime}(\omega)=B_{i j}^{\prime}(\omega) \equiv \frac{\partial}{\partial u_{k}} g_{i j}(\bar{u}) r_{k}^{\prime}(\omega), \quad 1 \leqslant l \leqslant n
$$

This is the precise meaning of Lemma 3.2.

Thus, a clear geometric distinction exists between elliptic and hyperbolic systems in terms of separation. For an elliptic system the wave cone is separated from the constitutive manifold $M$ by a hyperplane, namely the tangent space to $M$. For a hyperbolic system the wave cone intersects the tangent space and hence the manifold $M$ at least locally.

The following general result is due to Tartar [16]:

THEOREM 3.1. If the wave cone is separated from the tangent space in the sense of (3.1), then the Young measure $\nu$ associated with a weakly convergent sequence of functions $z^{\varepsilon}$ satisfying differential and algebraic constraints of the form (1.5) and (1.6) is a Dirac mass if the support of $\nu$ is sufficiently small.

As a corollary one may derive the following characterization of ellipticity in terms of compactness. 
THEOREM 3.2. A system of $n$ conservation laws in $n$ unknowns of the form (1.1) is elliptic if and only if all solution sequences with small oscillation are precompact in $L_{\text {loc }}^{1}$.

Tartar's proof of Theorem 3.1 will be discussed in $\S 8$. In $\S 6$ we shall present a proof of Theorem 3.2 in the special case where $m-1=2$ together with a generalization to the case of large oscillations for a certain class of systems. Our proof of Theorem 3.2 for systems with two independent variables uses weakly continuous quadratic forms. Tartar's proof of the general case makes use of weakly lower continuous quadratic forms.

4. The entropy condition and wave cone-manifold separation. We showed in $\S 3$ that the wave cone and constitutive manifold are not separated for hyperbolic systems. Separation may be recovered, however, in a higher dimensional space by introducing an entropy form. For simplicity we shall first consider hyperbolic systems in one space dimension,

$$
\partial_{t} u+\partial_{x} f(u)=0 ; \quad f: R^{n} \rightarrow R^{n} .
$$

We assume that $f$ is a smooth map whose Jacobian is diagonalizable at each state $u$, i.e. the eigenvalue problem $\{\lambda I-\nabla f(u)\} r=0$ admits $n$ independent eigenvectors $r_{j}=r_{j}(u)$ with eigenvalues $\lambda_{j}=\lambda_{j}(u)$. Additional structural hypotheses will be imposed on $f$ below.

It is well known that system (4.1) admits distributional solutions with discontinuities. The most primitive solutions are piecewise constant and consist of two states $v$ and $w$ separated in the $x-t$ plane by a line of discontinuity whose speed of propagation will be denoted by $\sigma$ :

$$
u(x, t)=\left\{\begin{array}{lc}
v & \text { if } x<\sigma t \\
w & \text { if } x>\sigma t
\end{array}\right\}
$$

The triple $(\sigma, v, w)$ satisfies the Rankine-Hugoniot relations

$$
\sigma(v-w)=f(v)-f(w)
$$

asserting the continuity of the normal component of the field $(u, f)$ across the line $x=\sigma t$. A result of Lax [12] states that if (4.1) is hyperbolic then for each state $v$ in $R^{n}$ there locally exists $n$ distinct curves in $R^{p}$ passing through $v$ with initial tangents $r_{j}(v)$ which consist of states $w$ that are connectable to $v$ through a discontinuity wave in the form (4.2)-(4.3). As a corollary we state the following result:

LEMMA 4.1. The system (4.1) is hyperbolic if and only if for all points a the wave cone $\Lambda_{a}$ intersects the constitutive manifold $M$ locally in $n$ independent curves in $R^{2 n}$ passing through $a$.

As a consequence, one may construct, for each state $v$ in $R^{n}$, a piecewise constant oscillating solution sequence of the form $u^{\varepsilon}=\varphi(x-\sigma t / \varepsilon)$ which converges weakly to a constant function whose value coincides with an arbitrary point on the line segment between $v$ and any specified point $w$ on the aformentioned wave curves constructed by Lax. Here $u^{\varepsilon}$ consists of an array of parallel discontinuities propagating with speed $\sigma$ and corresponds to the canonical codimension-one oscillating 
sequence discussed in $\$ 2$. The associated Young measure takes the form

$$
\nu=\theta \delta_{v}+(1-\theta) \delta_{w^{\prime}} \text {. }
$$

In order to discuss the effect of an entropy form on separation, we shall restrict our attention to strictly hyperbolic systems whose eigenvalues are genuinely nonlinear in the sense of Lax:

$$
r_{j} \cdot \nabla \lambda_{j}(u) \neq 0 \text {. }
$$

For genuinely nonlinear systems, the elementary two-state solutions (4.2) may be indexed by the associated eigenvalue $\lambda_{j}, 1 \leqslant j \leqslant n$, and then classified according to whether or not the associated characteristic curves

$$
d x / d t=\lambda_{j}(v) \text { and } \quad d x / d t=\lambda_{j}(w)
$$

both run into the line of discontinuities $x=\sigma t$ in the forward direction of time or both run away from the line of discontinuity in the forward direction. The former waves absorb entropy and are linearly stable. The latter waves radiate entropy and are linearly unstable, with respect to the forward time direction $[9,11]$.

The role of the classical entropy condition is to distinguish between these two possible situations for the purpose of creating a well-posed problem. In this connection we shall briefly recall the notion of generalized entropy in the sense of Lax [12].

Definition. A pair $(\eta, g)$ of real-valued maps on the state space $R^{n}$ is called an entropy pair for system (4.1) if all smooth solutions $u$ satisfy an additional conservation law of the form

$$
\partial_{t} \eta(u)+\partial_{x} q(u)=0 .
$$

The existence of an entropy pair is based upon a compatibility condition in $R^{n}$,

$$
\nabla \eta \nabla f=\nabla q,
$$

which may be derived by writing (4.1) and (4.5) in the quasilinear form

$$
\begin{gathered}
\partial_{t} u+\nabla f(u) \partial_{x} u=0, \\
\nabla \eta(u) \partial_{t} u+\nabla q(u) \partial_{x} u=0 .
\end{gathered}
$$

Substituting (4.7) into (4.8) yields

$$
\{-\nabla \eta \nabla f+\nabla q\} u_{x}=0 \text {. }
$$

In order for (4.9) to hold for all smooth solutions $u$, it is necessary that the coefficient vanish.

We remark that (4.6) is a formally overdetermined linear system of $n$ equations in two unknowns, $\eta$ and $q$, if $n>2$. Nevertheless, the systems of mechanics are naturally endowed with an entropy pair. In addition, the natural pair has the property that $\eta$ is strictly convex. Henceforth, we shall restrict our attention to strictly hyperbolic systems (4.1) which possess a strictly convex entropy pair. This class includes all systems of two equations, cf. [12]. In particular, the Lagrangian equations of elasticity (1.8) provides an example of special interest for which the entropy pair $\left(\eta_{1}, q_{1}\right)$ given by (1.10) plays a model role. We recall that if $\sigma^{\prime}>0$ then (1.8) is strictly hyperbolic and $\eta_{1}$ is strictly convex. 
With the aid of a single strictly convex entropy pair $(\eta, q)$ one may distinguish between discontinuities which absorb and radiate generalized entropy: The associated characteristics run into the shock in the forward direction of time if and only if the corresponding entropy field is nonpositive:

$$
\partial_{t} \eta(u)+\partial_{x} q(u) \leqslant 0 .
$$

We refer the reader to the article [12] of Lax for a general discussion of entropy. Here we shall merely state that it is traditional to impose an admissibility condition on weak solutions to systems of conservation laws in order to establish a well-posed problem.

Definition. An $L^{\infty}$ solution $u$ to $(4.1)$ is called admissible with respect to a strictly convex entropy pair $(\eta, q)$ if

$$
\partial_{t} \eta(u)+\partial_{x} q(u) \leqslant 0
$$

in the sense of distributions in $R^{2}$.

We remark that the property of admissibility is well defined: if $u$ satisfies (4.10) for one strictly convex entropy pair then it satisfies (4.10) for all strictly convex entropy pairs. The uniqueness and stability of admissible solutions to a scalar conservation law is well known $[10,18,20]$. For a partial result dealing with uniqueness of solutions of systems of two equations we refer the reader to [5].

CONJECTURE. If $u^{\varepsilon}$ is a sequence of admissible solutions to (4.1) with uniformly small oscillation then $u^{\varepsilon}$ is precompact in $L_{\text {loc }}^{1}$ provided that $(4.1)$ is strictly hyperbolic and genuinely nonlinear.

One might attempt to establish the validity of this conjecture by studying the separation of wave cone and manifold for the augmented system of $n+1$ equations

$$
\partial_{t} g_{0}(u)+\partial_{x} g_{1}(u)=\varphi
$$

defined by

$$
\begin{aligned}
& g_{0}=\{u, \eta(u)\}: R^{n} \rightarrow R^{n+1}, \\
& g_{1}=\{f(u), q(u)\}: R^{n} \rightarrow R^{n+1},
\end{aligned}
$$

that includes a single strictly convex entropy pair.

THEOREM 4.1. If the system (4.1) is strictly hyperbolic and genuinely nonlinear, then the wave cone and constitutive manifold of the augmented system (4.11) are separated in the sense of (2.17), at least locally.

Proof. We may rephrase the conclusion as follows: There exist no weak shock waves which conserve a strictly convex entropy field. In order to establish this fact let us fix a state $v$ in $R^{n}$ and consider any of the wave curves $w_{j}=w_{j}(\delta, v)$ which pass through $v$ and consist of states $w_{j}$ that satisfy the Rankine-Hugoniot relations

$$
\boldsymbol{\sigma}(\boldsymbol{\delta})\left\{w_{j}(\delta, v)-v\right\}=f\left\{w_{j}(\delta, v)\right\}-f(v) .
$$


A result of Lax [12] provides a Taylor expansion of the local entropy field associated with the discontinuity $\left(\sigma, w_{j}, v\right)$ in the form

$$
\begin{aligned}
\sigma\left\{\eta\left(w_{j}\right)-\eta(v)\right\}-q\left(w_{j}\right)+q(v) \\
=\text { const. } \nabla^{2} \eta(v)\left\{r_{j}(v), r_{j}(v)\right\} \delta^{3}+O\left(\delta^{4}\right)
\end{aligned}
$$

for small $\delta$. If $\lambda_{j}$ is genuinely nonlinear and $\eta$ is strictly convex then the coefficient of the cubic term (4.12) does not vanish.

REMARK 1 . The proof indicates that if system (4.1) is not genuinely nonlinear then it may not be possible to separate the wave cone and constitutive manifold by the introduction of just one strictly convex entropy pair. Simple example of nonseparation can be given in the setting of Lagrangian elasticity (1.8) when $\sigma$ has one inflection point, i.e. $\sigma^{\prime \prime}>0$ if $v>0$ while $\sigma^{\prime \prime}<0$ if $v<0$; $\mathrm{cf}$. $\S 5$. This property of nonseparation is directly connected with the manner in which entropy conditions must be formulated for nongenuinely nonlinear sytems.

REMARK 2. The introduction of a strictly convex entropy form does not separate the wave cone from the tangent space to the manifold. The tangent space $T_{a} M$ for (4.11) still intersects $\Lambda_{a}$ in $n$ directions since any solution $v$ of the linearized system

$$
\partial_{t} v+\nabla f(\bar{u}) \partial_{x} v=0
$$

associated with (4.1) is also a solution of the linearized system

$$
\partial_{t} v+\nabla f(\bar{u}) \partial_{x} v=0, \quad \nabla \eta(\bar{u}) \partial_{t} v+\nabla q(\bar{u}) \partial_{x} v=0
$$

associated with (4.11) due to the compatibility condition (4.6).

5. Lagrangian elasticity. In this section we shall be concerned with Tartar's conjecture 2.1 in the setting of Lagrangian elasticity. We shall formulate the main compactness result below and present the details of the proof in $\$ 9$. We first recall that the Lagrangian system (1.8) arises from a variational principle

$$
\delta \iint w_{t}^{2}-\Sigma\left(w_{x}\right) d x d t=0
$$

for the quasilinear wave equation

$$
w_{t t}-\sigma\left(w_{x}\right)_{x}=0
$$

for the displacement $w$, where $\Sigma$ is the primitive of the stress function $\sigma$. The first order system (1.8) is derived from (5.2) by introducing the velocity $u=w_{t}$ and the strain $v=w_{x}$. Since the integrand of (5.1) is invariant under temporal and spatial translations, all smooth solutions of (1.8) satisfy two additional conservation laws

$$
\partial_{t} \eta_{j}+\partial_{x} q_{j}=0
$$

where the pairs $\left(\eta_{j}, q_{j}\right)$ are given by (1.10) and (1.11).

We shall be concerned with the structure in state space of the augmented system (1.9) of four equations. Here, the ambient state space $R^{N}$ has dimension eight. The constitutive manifold $M$ has dimension two and is conveniently parametrized by 
$(u, v): M=\left\{w_{i j} \in R^{8}: w_{i j}=g_{i j}(u, v), 1 \leqslant i \leqslant 4,0 \leqslant j \leqslant 1\right\}$ where

$$
\begin{aligned}
& g_{0}=\left\{v, u, \eta_{1}(u, v), \eta_{2}(u, v)\right\}, \\
& g_{1}=\left\{-u,-\sigma(v), q_{1}(u, v), q_{2}(u, v)\right\} .
\end{aligned}
$$

THEOREM 5.1. Suppose $\sigma^{\prime}>0$ and $\sigma^{\prime \prime} \neq 0$. If $\left(u^{\varepsilon}, v^{\varepsilon}\right)$ is a sequence of functions with small oscillation such that each of the sequences of distributions

$$
\sum_{j=0}^{1} \partial_{j} g_{i j}\left(u^{\varepsilon}, v^{\varepsilon}\right), \quad 1 \leqslant i \leqslant 4,
$$

lies in a compact subset of $W_{\mathrm{loc}}^{-1.2}\left(R^{2}\right)$, then there exists a subsequence of $\left(u^{\varepsilon}, v^{\varepsilon}\right)$ which converges in the strong topology of $L_{\mathrm{loc}}^{1}\left(R^{2}\right)$.

We shall show that the augmented system (1.9) has a fourth order locally coercive structure in the state space $R^{8}$ which has the effect of reducing the Young measure associated with $\left(u^{\varepsilon}, v^{\varepsilon}\right)$ to a Dirac mass. This fourth order structure provides an interesting contrast with the second order locally coercive structure associated with elliptic systems. For this reason we shall consider elliptic systems in the next section.

THE NONCONVEX CASE. It would be interesting to consider the compactness of motions of materials with a nonconvex equation of state. It turns out that if the system (1.8) is not genuinely nonlinear, i.e. $\sigma$ is neither strictly convex nor strictly concave, then the wave cone associated with the doubly augmented system (1.9) of four equations is not separated from $M$. This fact may be easily verified using the calculations given in $\$ 10$. We conjecture that the wave cone associated with a system of conservation laws that is not genuinely nonlinear cannot be separated from the constitutive manifold through the introduction of any finite number of entropy forms.

6. The Div-Curl Lemma and elliptic systems. In this section we shall establish the compactness of solutions with small oscillation to general elliptic systems in the plane and the compactness of solutions with large oscillations for a special class of elliptic systems in the plane. One of the main tools is a basic lemma that provides a classification of weakly continuous maps $g=g(w, z)$ acting on sequences of vector fields $w^{\varepsilon}$ and $z^{\varepsilon}$ such that

$$
\operatorname{div} w^{\varepsilon} \equiv \sum_{j=0}^{m-1} \partial_{j} w_{j}^{\varepsilon} \quad \text { and } \quad \operatorname{curl} z^{\varepsilon} \equiv \partial_{j} w_{k}^{\varepsilon}-\partial_{k} w_{j}^{\varepsilon}
$$

lie in a compact subset of $W_{\text {loc }}^{-1,2}\left(R^{m}\right)$. A result of Tartar and Murat $[13,16]$ asserts that $g$ is weakly continuous if and only if $g$ coincides with the inner product modulo an affine function, and that $g$ is weakly lower continuous, i.e.

$$
w-\lim g\left(w^{\varepsilon}, z^{\varepsilon}\right) \geqslant g\left(w-\lim w^{\varepsilon}, w-\lim z^{\varepsilon}\right)
$$

if $g$ coincides with the inner product modulo a convex function, i.e.

$$
g=\sum_{j=0}^{m-1} w_{j} z_{j}+c
$$


where $c$ is convex. We remark that, in general, none of the individual terms in the summation (6.2) is continuous in the weak topology under the hypothesis of the compactness of (6.1). However, there exists a compensation between the terms which allows for the weak continuity of the sum. This lemma has an interesting interpretation in the setting of electrostatics, cf. [15]. Using this Div-Curl Lemma establish the following result:

THEOREM 6.1. Suppose that

$$
\sum_{j=0}^{1} \partial_{j} g_{j}(u)=0, \quad g_{j}: R^{p} \rightarrow R^{p},
$$

is an elliptic system. If $u^{\varepsilon}$ is a sequence of functions with small oscillation such that each of the sequences of distributions

$$
\sum_{j=0}^{1} \partial_{j} g_{i j}\left(u^{\varepsilon}\right), \quad 1 \leqslant i \leqslant p,
$$

lies in a compact subset of $W_{\mathrm{loc}}^{-1,2}\left(R^{2}\right)$, then there exists a subsequence which converges in the strong topology.

Several preliminary remarks are in order. Suppose that $\left(\eta_{j}, q_{j}\right)$ are two entropy pairs for a general system of $p$ conservation laws in two independent variables of the form (6.3). Assume, for the moment, that no information concerning the type of the system is given. Let $u^{\varepsilon}$ be a sequence of solutions to (6.3) such that both of the entropy fields

$$
\partial_{t} \eta_{j}\left(u^{\varepsilon}\right)+\partial_{x} q_{j}\left(u^{\varepsilon}\right)
$$

lie in a compact subset of $W_{\mathrm{loc}}^{-1,2}\left(R^{2}\right)$. As a consequence of the orthogonal duality between the divergence and curl operators in two dimensions, namely

$$
\operatorname{div} h=\partial_{1} h_{1}+\partial_{2} h_{2}=\operatorname{curl}\left(h_{2},-h_{1}\right)=\operatorname{curl} h^{\perp},
$$

the fields

$$
\operatorname{div}\left\{\eta_{1}\left(u^{\varepsilon}\right), q_{1}\left(u^{\varepsilon}\right)\right\} \quad \text { and } \operatorname{curl}\left\{-q_{2}\left(u^{\varepsilon}\right), \eta_{2}\left(u^{\varepsilon}\right)\right\}
$$

are compact. Thus, we may deduce the continuity of the inner product after passing to an appropriate subsequence

$$
\begin{aligned}
& w-\lim \eta_{1}^{\varepsilon} q_{2}^{\varepsilon}-\eta_{2}^{\varepsilon} q_{1}^{\varepsilon}=\eta_{1}^{0} q_{2}^{0}-\eta_{2}^{0} q_{1}^{0} \\
& \quad \text { where } \eta_{j}^{0}=w-\lim \eta_{j}^{\varepsilon} \text { and } q_{j}^{0}=w-\lim q_{j}^{\varepsilon} .
\end{aligned}
$$

Equation (6.4) may be rewritten in terms of the Young measure $\nu_{y}$ associated with $u^{\varepsilon}$ in the following way:

$$
\left\langle\nu, \eta_{1} q_{2}-\eta_{2} q_{1}\right\rangle=\left\langle\nu, \eta_{1}\right\rangle\left\langle\nu, q_{2}\right\rangle-\left\langle\nu, \eta_{2}\right\rangle\left\langle\nu, q_{1}\right\rangle \text {. }
$$

The left-hand side of (6.5) represents the weak limit of the inner product while the right-hand side represents the inner product of the weak limits. We emphasize that (6.5) holds for all entropy pairs such that the corresponding entropy fields (6.4) are compact. The commutativity relation (6.6) is Tartar's functional equation described in the introduction; it is satisfied by $\nu_{y}$ for almost all $y$ in $R^{2}$. 
Remarks. For a general system of conservation laws (6.3) the compatibility condition between a generalized entropy $\eta$ and its associated generalized entropy flux $q$ forms a set of $p$ differential equations in two unknowns, $\eta$ and $q$, which retains the type of the parent system (6.3). Independently of the type of system (6.2) there always exists a small class of entropy pairs

$$
\eta=\sum_{i=1}^{p} \alpha_{i} g_{i 1}, \quad q=\sum_{i=1}^{p} \alpha_{i} g_{i 2},
$$

formed from linear combinations of the equations which compose the system. Here $\alpha$ is an arbitrary vector in $R^{p}$. Clearly any smooth solution $u$ of (6.3) satisfies

$$
\partial_{t} \eta(u)+\partial_{x} q(u)=0
$$

where $\eta$ and $q$ are given by (6.7). For elliptic systems in the plane one may establish compactness by applying the Div-Curl Lemma to entropy pairs formed from linear combinations of the flux functions $g_{i j}$.

THEOREM 6.2. Suppose that $\nu$ is an arbitrary probability measure which satisfies the commutativity relation (6.6) when applied to entropy pairs of the form (6.7). If (6.3) is elliptic and if the support of $\nu$ is sufficiently small then $\nu$ is a Dirac mass.

Proof. Let us fix the center of mass $\bar{u}=\langle\nu, \lambda\rangle$ and rewrite the commutativity relation (6.6) in the form

$$
\left\langle\nu, \delta \eta_{1} \delta q_{2}-\delta \eta_{2} \delta q_{1}\right\rangle=\left\langle\nu, Q \eta_{1}\right\rangle\left\langle\nu, Q q_{2}\right\rangle-\left\langle\nu, Q \eta_{2}\right\rangle\left\langle\nu, Q q_{1}\right\rangle
$$

in terms of the linear and quadratic parts of $\eta$ and $q$ at $\bar{u}$ :

$$
\begin{array}{llrl}
\delta \eta & =\eta(u)-\eta(\bar{u}), & & Q \eta=\eta(u)-\eta(\bar{u})-\nabla \eta(\bar{u})(u-\bar{u}), \\
\delta q=q(u)-q(\bar{u}), & & Q q=q(u)-q(\bar{u})-\nabla q(\bar{u})(u-\bar{u}) .
\end{array}
$$

Here we have used the fact that the expected value of the linear and quadratic parts of any state variable coincide since $\bar{u}$ is the center of mass $\langle\nu, \delta \varphi\rangle=\langle\nu, Q \varphi\rangle$.

We ask when it is possible to choose a finite set of $s$ entropy pairs such that the sum of the integrands on the left-hand side of (6.6) is locally positive definite, i.e.

$$
F \equiv \sum_{j, k=1}^{s} \delta \eta_{j} \delta q_{k}-\delta \eta_{k} \delta q_{j} \geqslant \text { const. }|u-\bar{u}|^{2} .
$$

If (6.10) can be achieved in a ball $B_{r}$, a radius $r$ centered at $\vec{u}$, and if the support of $\nu$ is contained in $B_{r}$, then (6.9) implies that

$$
\left\langle\nu,|u-\bar{u}|^{2}\right\rangle \leqslant \text { const. }\left\langle\nu,|u-\bar{u}|^{2}\right\rangle^{2},
$$

and hence that $\nu$ is a Dirac mass at $\bar{u}$ if $r$ is sufficiently small. It turns out that (6.10) can be achieved if and only if (6.3) is elliptic. If (6.3) is elliptic, then (6.10) can be achieved with entropy pairs of the form (6.7).

Without loss of generality we shall consider systems where $g_{0}$ is the identity map. Taking

$$
\eta_{k}=\alpha_{k} \cdot(u-\bar{u}), \quad q_{k}=\alpha_{k} \cdot\{g(u)-g(\bar{u})\}
$$


yields

$$
F=\sum_{k, j}\left(\alpha_{k} \otimes \alpha_{j}-\alpha_{j} \otimes \alpha_{k}\right) \nabla g_{1}(\bar{u})(u-\bar{u})^{2}+O(u-\bar{u})^{2}
$$

where the tensor product of two vectors $\alpha$ and $\beta$ in $R^{p}$ is defined in the standard fashion as the $p \times p$ matrix

$$
\{\alpha \otimes \beta\}_{i j}=\alpha^{i} \beta^{j}
$$

We observe that

$$
\alpha \vee \beta \equiv \alpha \otimes \beta-\beta \otimes \alpha
$$

is a basic antisymmetric matrix: Any antisymmetric matrix $A$ can be expressed as a finite sum of antisymmetric parts of dyadic products:

$$
A=\sum_{j} \alpha_{j} \vee \beta_{j} .
$$

Thus we are lead to the question of whether or not there exists an antisymmetric matrix $A$ such that the quadratic form associated with matrix

$$
S \equiv A G+(A G)^{t} ; \quad G=\nabla g_{1}(\bar{u})
$$

is positive definite. The answer is yes if the linearized system corresponding to (6.3) has no real characteristic hypersurfaces, i.e. if $G$ is an elliptic matrix in the sense that it has no real spectrum

$$
\sigma(G) \cap R=\varnothing .
$$

The proof of Theorem 6.2 follows from the next proposition.

Proposition. If $G$ is an arbitrary elliptic matrix in the sense of (6.11), then there exists an antisymmetric matrix $A$ such that (6.10) is positive definite.

Proof. We note that $p$ is even if (6.3) is elliptic. In the case $p=2$, a simple computation shows that the characteristic polynomial of a $2 \times 2$ matrix $G$ has purely imaginary roots if and only if the symmetric part $S$ of the matrix $A G$ is definite, where $A$ is the elementary antisymmetric matrix

$$
\left(\begin{array}{rr}
0 & 1 \\
-1 & 0
\end{array}\right)
$$

The general case is easily reduced to the case $p=2$ if $G$ is diagonalizable over the complex field, i.e. if the real Jordan normal form of $G$ is a $2 \times 2$ block-diagonal matrix. To see this, we remark that a symmetric matrix $C$ is positive definite if and only if there exists an invertible matrix $P$ such that the conjugate matrix $P^{t} C P$ is positive definite. Thus, $S$ is positive if and only if there exists an invertible $P$ such that

$$
\tilde{S}=\tilde{A} \tilde{G}+(\tilde{A} \tilde{G})^{t}>0, \quad \text { where } \tilde{A}=P^{t} A P \text { and } \tilde{G}=P^{-1} G P
$$

since

$$
\tilde{S}=P^{t} S P
$$


Hence, if $G$ is similar to a $2 \times 2$ block-diagonal matrix $\tilde{G}$, then we simply take $\tilde{A}$ to be a $2 \times 2$ block-diagonal antisymmetric matrix each of whose diagonal blocks equals (6.12) or its negative. The desired matrix $A$ is the conjugate of $\tilde{A}$ given by (6.13). The most general case is handled by expressing $G$ in $2 \times 2$ Jordan block form and selecting a basis so that the blocks off the diagonal are small. The details are omitted.

LARGE OSCILLATION. Several situations arise in which the proof of the local result above extends to the case where the support of the Young measure is an arbitrary compact set. For example, let us consider $2 \times 2$ elliptic systems of the form

$$
\begin{aligned}
& \partial_{0} v-\partial_{1} u=0, \quad \sigma^{\prime}(v)<0, \\
& \partial_{0} u-\partial_{1} \sigma=0 .
\end{aligned}
$$

This class includes the small disturbance equations restricted to subsonic flow. Using the entropy pairs

$$
\left(\eta_{1}, q_{1}\right)=(u,-\sigma) \text { and }\left(\eta_{2}, q_{2}\right)=(v,-u)
$$

the commutativity relation (6.9) takes the form

$$
\left\langle\nu,(u-\bar{u})^{2}-\{\sigma(v)-\sigma(\bar{v})\}\{v-\bar{v}\}\right\rangle=0,
$$

since $\eta_{j}$ is linear. It follows immediately from (6.14) that $\nu$ is concentrated at $(\bar{u}, \bar{v})$ if $\sigma^{\prime}<0$.

A generalization of this example deals with elliptic systems of the form

$$
\partial_{0} u+B \partial_{x} f(u)=0
$$

where $B$ is an antisymmetric matrix and $f$ is a strictly monotone map on $R^{p}$, i.e.

$$
\{f(u)-f(v), u-v\}>0,
$$

for all $u \neq v$ in $R^{p}$. Strict monotonicity means that the symmetric part of the Jacobian of $f$ is positive. We may therefore take $A$ to be the inverse of $\beta$.

REMARKS. There are several senses in which an elliptic system exhibits a coercive structure in physical space and function space associated with a gain of derivatives in the scale of Sobolev spaces, etc. The inequality (6.10) may be regarded as a statement of coercive structure in the state space. It motivates the search for coercive structure for hyperbolic systems in state space.

7. Polyconvexity and compactness. We shall be concerned with the equations of elastostatics for hyperelastic materials.

$$
\frac{\partial}{\partial X_{\alpha}}\left(\frac{\partial W}{\partial F_{\alpha}^{i}}\right)=0, \quad 1 \leqslant i \leqslant n .
$$

The displacement of a material contained in a reference configuration $\Omega$ of $R^{n}$ and coordinatized by $X_{\alpha}$ is described by functions

$$
x^{i}=x^{i}\left(X_{\alpha}\right)
$$

which determine a stress tensor $F_{\alpha}^{i}=\partial x^{i} / \partial X_{\alpha}$. The material properties are characterized by a stored energy function $W(F)$ which acts as a potential for the stress matrix $T_{\alpha}^{i}=\partial W / \partial F_{\alpha}^{i}$. The second order system of $n$ equations for the displacement 
functions (7.2) may be recast as a first order system of $2 n$ equations for the deformation gradient $F_{\alpha}^{i}$,

$$
\begin{gathered}
\operatorname{curl} F_{\alpha}^{i}=0, \\
\operatorname{div}\left(\partial W / \partial F_{\alpha}^{i}\right)=0,
\end{gathered}
$$

that expresses the conservation of mass (7.3) and momentum (7.4). As an immediate consequence of the Div-Curl Lemma, one may establish the following compactness theorem:

THEOREM 7.1. If $W$ is strictly convex and if $F^{\varepsilon}$ is a uniformly bounded solution sequence to (7.3)-(7.4) then $F^{\varepsilon}$ contains a subsequence that converges in the strong topology of $L_{\text {loc }}^{1}$.

Proof. Let $\nu$ denote the Young measure associated with a weakly convergent subsequence of $F^{\varepsilon}$. The Div-Curl Lemma implies that

$$
\sum_{i, \alpha}\left\langle\nu, \frac{\partial W}{\partial F_{\alpha}^{i}} F_{\alpha}^{i}\right\rangle=\sum_{i, \alpha}\left\langle\nu, \frac{\partial W}{\partial F_{\alpha}^{i}}\right\rangle\left\langle\nu, F_{\alpha}^{i}\right\rangle
$$

or equivalently

$$
\langle\nu, E(F, \bar{F})\rangle=0
$$

where $E$ denotes the elliptic form at the center of mass of $\nu$ :

$$
E(F, \bar{F})=\sum_{i, \alpha}\left\{\frac{\partial W}{\partial F_{\alpha}^{i}}(F)-\frac{\partial W}{\partial F_{\alpha}^{i}}(\bar{F})\right\}\left\{F_{\alpha}^{i}-\bar{F}_{\alpha}^{i}\right\}, \quad \bar{F}_{\alpha}^{i}=\left\langle\nu, F_{\alpha}^{i}\right\rangle .
$$

If $W$ is strictly convex then its gradient is strictly monotone, i.e. $E$ is positive for all $F$ and $\bar{F}$ such that $F \neq \bar{F}$, and (7.5) implies that $\nu$ is concentrated at $\bar{F}$.

REMARK 1. The theorem can also be easily established with Minty's device.

REMARK 2. It is well known that global convexity of $W$ is not physically reasonable. For example, it is not consistent simultaneously with frame indifference and blow-up as the determinant of $F$ vanishes. However, $W$ may be convex on a fairly large region. The class of polyconvex materials, J. Ball [2], C. B. Morrey, Jr. [21], provides a setting in which frame indifference and physical growth conditions are satisfied. We refer the reader to the articles of J. Ball [1, 2] for an introduction to elasticity in the context of the calculus of variations and for a discussion of polyconvexity. The main point of this section is that the compactness of elastostatic solutions for polyconvex materials is a direct consequence of the Div-Curl Lemma and the weak continuity of the subdeterminants of a Jacobian matrix. The relevance of the weak continuity of subdeterminants for elasticity was first recognized by $\mathrm{J}$. Ball [2]. In terms of the Young measure, weak continuity of the determinant of $F_{\alpha}^{i}$ and of the determinants of the matrices formed by deleting an equal number of rows and columns from $F$ may be expressed by the statement that

$$
\left\langle\nu, \text { subdet } F_{\alpha}^{i}\right\rangle=\operatorname{subdet}\left\langle\nu, F_{\alpha}^{i}\right\rangle \text {. }
$$


THEOREM 7.2. If $W$ is strictly polyconvex and $F^{\varepsilon}$ is a solution sequence of (7.3)-(7.4) with small oscillation, then there exists a subsequence which converges in the strong topology of $L_{\text {loc }}^{1}$.

This theorem is an immediate corollary of the following lemma:

LEMMA 7.1. If $W$ is strictly polyconvex and if $\nu$ is an arbitrary probability measure satisfying (7.5) and (7.7) where $\bar{F}$ denotes the center of mass, then $\nu$ is concentrated at $\bar{F}$ if the diameter of its support is sufficiently small.

We recall that by definition $W$ is polyconvex if it can be represented as a convex function $h$ of the matrix $F$, the adjugate of $F$ and the determinant of $F$ :

$$
W(F)=h(F, \text { ad } F, \operatorname{det} F) .
$$

Here ad $F$ denotes the transposed $n \times n$ matrix of cofactors of $F$. The stored-energy function $W$ will be referred to as strictly polyconvex if $h$ is strictly convex. The proof of Lemma 7.1 is based upon the observation that the elliptic form $E$ differs locally from the quadratic part of $h$ by a form with zero expected value, i.e.

$$
E=N(F, \bar{F})+Q h(z, \bar{z})+O(F-\bar{F})^{3}
$$

where

$$
\begin{gathered}
Q h=\{\nabla h(z)-\nabla h(\bar{z}), z-\bar{z}\}, \\
z=(F, A, \tau) ; \quad A=\operatorname{adt} F, \quad \tau=\operatorname{det} F
\end{gathered}
$$

and

$$
\langle\nu, N\rangle=0 \text {. }
$$

In equation (7.9) the brackets denote the standard Euclidean inner product. It is somewhat more convenient technically to work with the matrix of cofactors of $F$, adt $F$, than with the transposed matrix of cofactors ad $F$. If $h$ is a (strictly) convex function of $z$ then $Q h$ is (positive) nonnegative. If $W$ is strictly convex then the conditions

$$
\langle\nu, E\rangle=0, \quad\langle\nu, N\rangle=0, \quad Q h \geqslant \text { const. }|z-\bar{z}|^{2},
$$

together with (7.8), imply that $\nu$ is concentrated at $\bar{F}$ if its support is sufficiently small.

REMARK. The fact that $N$ has zero expected value is due to the weak continuity of (7.7) of subdeterminants. The equation (7.7) expresses the fact that the only weakly continuous maps in the presence of differential constraints of the form (7.3) are null-Lagrangian, i.e. maps for which the associated Euler-Lagrange equation holds identically. We refer the reader to $[2,3]$ for a general discussion of null-Lagrangians and their relevance to the existence of minimizers for the stored-energy functional.

PROPOSITION 7.1. The form $E$ differs locally from a null-Lagrangian form $N$ by the quadratic part of $h$ in the sense of (7.8).

PROOF. The verification of (7.8) depends on a straightforward Taylor expansion. We shall consider the case $n=3$ and write the derivative of $W$ in the form

$$
d W=\left[\frac{\partial h}{\partial F}, \frac{\partial h}{\partial A} \frac{\partial A}{\partial F}, \frac{\partial g}{\partial \tau} \frac{\partial \tau}{\partial F}\right] .
$$


We recall that the derivative of a determinant is represented by the matrix of cofactors

$$
d \tau(F)=A(F) ; \quad A_{\alpha}^{i}(F)=\operatorname{det} m_{\alpha}^{i}(F)
$$

where $m_{\alpha}^{i}$ is the $2 \times 2$ matrix obtained from $F$ by deleting row $i$ and column $\alpha$. Thus, the derivative of $W$ at $F$ in the direction of $\tilde{F}$ may be written in form $d W(F, \tilde{F})=\left\{\frac{\partial h}{\partial F}(z), \tilde{F}\right\}+\sum_{i, \alpha} \frac{\partial h}{\partial A_{\alpha}^{i}}(z)\left\{\operatorname{adt} m_{\alpha}^{i}(F), m_{\alpha}^{i}(\tilde{F})\right\}+\frac{\partial g}{\partial \tau}(z)\{A, \tilde{F}\}$.

Similarly,

$$
\begin{aligned}
E(F, \bar{F})= & \left\{\frac{\partial h}{\partial F}(z)-\frac{\partial h}{\partial F}(\bar{z}), F-\bar{F}\right\} \\
& +\sum_{i, \alpha}\left\{\frac{\partial h}{\partial A_{\alpha}^{i}}(z) \text { adt } m_{\alpha}^{i}(F)-\frac{\partial h}{\partial A_{\alpha}^{i}}(\bar{z}) \text { adt } m_{\alpha}^{i}(\bar{F}), m_{\alpha}^{i}(F)-m_{\alpha}^{i}(\bar{F})\right\} \\
& +\left\{\frac{\partial h}{\partial t}(z) A-\frac{\partial h}{\partial t}(\bar{z}) \bar{A}, F-\bar{F}\right\} .
\end{aligned}
$$

We wish to compare $E$ with the quadratic part of $h$, i.e. with

$$
\begin{aligned}
Q h= & \left\{\frac{\partial h}{\partial F}(z)-\frac{\partial h}{\partial F}(\bar{z}), F-\bar{F}\right\}+\left\{\frac{\partial h}{\partial A}(z)-\frac{\partial h}{\partial A}(\bar{z}), A-\bar{A}\right\} \\
& +\left(\frac{\partial h}{\partial \tau}(z)-\frac{\partial h}{\partial \tau}(\bar{z})\right)(\tau-\bar{\tau}) .
\end{aligned}
$$

The difference between (7.11) and (7.12) may be analyzed as follows: Write the third term of (7.11) as

$$
Y=\frac{\partial h}{\partial \tau}(\bar{z})\{A-\bar{A}, F-\bar{F}\}+\left(\frac{\partial h}{\partial \tau}(z)-\frac{\partial h}{\partial \tau}(\bar{z})\right)\{A, F-\bar{F}\} .
$$

The relation (7.10) implies that

$$
\operatorname{det} \bar{F}=\operatorname{det} F+\{A, F-\bar{F}\}+O(F-\bar{F})^{2},
$$

and therefore that

$$
\{A, F-\bar{F}\}=\operatorname{det} F-\operatorname{det} \bar{F}+O(F-\bar{F})^{2} .
$$

Thus $Y$ may be written in the form

$$
\text { (7.13) } Y=\frac{\partial h}{\partial \tau}(\bar{z})\{A-\bar{A}, F-\bar{F}\}+\left(\frac{\partial h}{\partial \tau}(z)-\frac{\partial h}{\partial \tau}(\bar{z}), \tau-\bar{\tau}\right)+O(F-\bar{F})^{3} \text {. }
$$

The first term on the right-hand side of (7.13) contributes by definition to $N$. The second term is contained in (7.12) and the third is a cubic residual.

Next let us analyze the second term in (7.11) and write it in the form

$$
Z=\frac{\partial h}{\partial A}(\bar{z})\{\text { adt } M-\text { adt } \bar{M}, M-\bar{M}\}+\left\{\frac{\partial h}{\partial A}(z)-\frac{\partial h}{\partial A}(\bar{z}) \text { adt } M, M-\bar{M}\right\}
$$

where $M(F)$ denotes an array of $2 \times 2$ matrices $m_{\alpha}^{i}(F)$ and $\bar{M}=M(\bar{F})$. We observe that

$$
\operatorname{adt} M_{\alpha}^{i}\left(M_{\alpha}^{i}-\bar{M}_{\alpha}^{i}\right)=\operatorname{det} M_{\alpha}^{i}-\operatorname{det} \bar{M}_{\alpha}^{i}+O(M-\bar{M})^{2} .
$$


Since by definition the determinant of $M_{\alpha}^{i}$ is $A_{\alpha}^{i}$, we have

$$
\text { adt } M_{\alpha}^{i}\left(M_{\alpha}^{i}-\bar{M}_{\alpha}^{i}\right)=A_{\alpha}^{i}-\bar{A}_{\alpha}^{i}+O(F-\bar{F})^{2},
$$

and

$$
\begin{aligned}
Z= & \frac{\partial h}{\partial A}(\bar{z})\{\text { adt } M-\operatorname{adt} \bar{M}, M-\bar{M}\} \\
& +\left\{\frac{\partial h}{\partial A}(z)-\frac{\partial h}{\partial A}(\bar{z}), A-\bar{A}\right\}+O(F-\bar{F})^{3}
\end{aligned}
$$

As before, the first terms in (7.14) contribute to $N$. The second is contained in (7.12) and the third is a cubic residual. We therefore obtain the desired decomposition (7.8) with

$$
N=\frac{\partial h}{\partial \tau}(\bar{z})\{A-\bar{A}, F-\bar{F}\}+\frac{\partial h}{\partial A}(\bar{z})\{\operatorname{adt} M-\operatorname{adt} \bar{M}, M-\bar{M}\}
$$

The proof of Proposition 7.1 is complete.

It remains only to observe that the expected value of $N$ is zero. We note that the first term of (7.15) has zero expected value:

$$
\begin{aligned}
\left\langle\nu, \sum\left(A_{\alpha}^{i}-\bar{A}_{\alpha}^{i}\right)\left(F_{\alpha}^{i}-\bar{F}_{\alpha}^{i}\right)\right\rangle & =\left\langle\nu, \sum\left(A_{\alpha}^{i}-\vec{A}_{\alpha}^{i}\right) F_{\alpha}^{i}\right\rangle \\
& =\sum\left\langle\nu, A_{\alpha}^{i} F_{\alpha}^{i}\right\rangle-\sum A_{\alpha}^{i}(\bar{F}) \bar{F}_{\alpha}^{i} \\
& =3\langle\nu, \operatorname{det} F\rangle-3 \operatorname{det}\langle\nu, F\rangle=0
\end{aligned}
$$

since, in general, $\{A, F\}=n \operatorname{det} F$. The second term of (7.15) is treated similarly.

REMARK. It would be interesting to determine the size of the set on which $E-N$ has a distinguished sign. It appears that for a typical Neo-Hookian material $E-N$ does not have a distinguished sign globally.

8. Compactness with maximal separation of wave cone and manifold. In this section we shall discuss Theorem 3.1 asserting the reduction of the Young measure to a point mass if the tangent space is separated from the wave cone in the sense of (3.1). We shall emphasize those features which are relevant to our treatment of dynamic elasticity in $\$ 9$.

Consider the structure presented by (2.1), (2.7), (2.3) and assume that $M$ is an $n$-dimensional manifold coordinatized by $g: R^{n} \rightarrow R^{n}$. Specifically, assume that the sequence $z^{\varepsilon}$ takes the form

$$
z^{\varepsilon}=g\left(u^{\varepsilon}\right)
$$

where $u^{\varepsilon}: R^{m} \rightarrow R^{n}$ converges weakly and satisfies $\left|u^{\varepsilon}-u^{*}\right|_{\infty} \leqslant r$, where $u^{*}$ is a fixed state in $R^{n}$ and $r$ is small. It will be shown that the Young measure $\sigma_{y}$ associated with the sequence $u^{\varepsilon}$ is a Dirac mass for almost all $y$ and hence that the Young measure $\nu_{y}$ associated with the sequence $z^{\varepsilon}$ is a Dirac mass. Equation (8.1) implies that $\nu$ is the image of $\sigma$ under $g$, i.e.

$$
\langle\nu, h(\lambda)\rangle=\langle\sigma, h \circ g(u)\rangle
$$

for all $h$. Here $\lambda$ and $u$ denote the generic variables of $R^{N}$ and $R^{n}$, respectively. 
The strategy for proving Theorem 3.1 is to construct a quadratic map $Q$ on the ambient space $R^{N}$ which is convex in the directions of the wave cone $\Lambda$ associated with (2.2) and concave in the directions of the tangent space to $M$. The compensated compactness Theorem 2.2 for quadratic maps implies that $Q$ is weakly lower continuous and therefore satisfies a Jensen-type inequality (2.16) with respect to $\nu$. The subsequent restriction to $M$ induces a reverse Jensen inequality that reduces $\nu$ to a Dirac mass.

Proof of Theorem 3.1. Fix $y$ and let $b$ denote the image of the center of mass of $\sigma_{y}$ under $g$ :

$$
b=g\left(\left\langle\sigma_{y}, u\right\rangle\right) .
$$

Define $Q(\lambda)$ as the square of the Euclidean distance from $\lambda$ to the (linear) tangent space $T M$ of $M$ at $b$ minus a small factor of the square of the distance to $b$ :

$$
Q(\lambda)=\operatorname{dist}^{2}(\lambda-b, T M)-\delta|\lambda-b|^{2} \text {. }
$$

The separation hypothesis (3.1) implies that $Q$ is convex in the directions of $\Lambda$ if $\delta$ is small and therefore that (2.16) holds. Using (8.2) we may rewrite (2.16) in terms of $\sigma$ as follows:

$$
Q(\langle\sigma, g(u)\rangle) \leqslant\langle\sigma, Q(g(u))\rangle \text {. }
$$

Observe that the left-hand side of (8.5) is fourth order with respect to the distance from $u$ to the center of mass $\bar{u}$ of $\sigma$. We note that

$$
\bar{g}-b=\left\langle\sigma, O(u-\bar{u})^{2}\right\rangle, \quad \text { where } \bar{g} \equiv\langle\sigma, g(u)\rangle,
$$

since

$$
\bar{g}=\langle\sigma, g(u)-g(\bar{u})\rangle+g(\bar{u})=\langle\sigma, g(u)-g(\bar{u})+\nabla g(\bar{u})(u-\bar{u})\rangle+b .
$$

Thus, the left-hand side of $(8.5)$ is fourth order:

$$
Q(\bar{g})=\operatorname{dist}^{2}(\bar{g}-b, T M)-\delta|\bar{g}-b|^{2}=O\left(\left\langle\sigma,|u-\bar{u}|^{2}\right\rangle\right)^{2}
$$

Second, observe that $Q$ is locally negative definite on $M$, i.e.

$$
\begin{aligned}
Q(g(u)) & =\operatorname{dist}^{2}(g(u)-b, T M)-\delta|g(u)-g(\bar{u})|^{2} \\
& \leqslant \text { const. }|u-\bar{u}|^{4}-\delta|g(u)-g(\bar{u})|^{2},
\end{aligned}
$$

if $u$ lies near $\bar{u}$. Indeed, (8.7) follows from the fact that $g-b$ is quadratic modulo a tangent vector $\tau$, i.e.

$$
\begin{aligned}
g(u)-b & =\{g(u)-g(\bar{u})-\nabla g(\bar{u})(u-\bar{u})\}+\nabla g(\bar{u})(u-\bar{u}) \\
& =O(u-\bar{u})^{2}+\tau .
\end{aligned}
$$

Thus,

$$
\operatorname{dist}(g(u)-b, T M) \leqslant \text { const. }|u-\bar{u}|^{2} .
$$

Since $g$ is a coordinate map, we conclude that

$$
Q(g(u)) \leqslant- \text { const. }|u-\bar{u}|^{2}
$$


if $r$ is small and hence that

$$
\left\langle\sigma,|u-\bar{u}|^{2}\right\rangle \leqslant \text { const. }\left\langle\sigma,|u-\bar{u}|^{2}\right\rangle^{2} .
$$

Thus $\sigma$ is concentrated at $\bar{u}$.

REMARK 1. It would have been sufficient to employ a weaker upper bound of the form

$$
Q(g(u)) \leqslant- \text { const. }|u-\bar{u}|^{s}
$$

if $s<4$. More generally a bound of the form (8.8) with $s=4$ leads to an inequality

$$
\left\langle\sigma,|u-\bar{u}|^{2}\right\rangle^{2} \leqslant \theta\left\langle\sigma,|u-\bar{u}|^{4}\right\rangle
$$

which induces a reversal of Jensen's inequality if $\theta \leqslant 1$. The only probability measure which satisfies a reverse Jensen inequality is a Dirac mass.

REMARK 2. For hyperbolic systems there does not exist a separating quadratic form of the type $Q$ constructed above. At best, one would hope that, in the hyperbolic context, a quadratic form would exist which is nonnegative on $\Lambda$ and locally fourth order nonpositive on $M$. We shall see in the next section that a favorable fourth ordeer structure exists for Lagrangian elasticity in one space dimension, although one which is not as simple as (8.9).

REMARK 3. There is no intrinsic reason for restricting attention to quadratic maps. When additional information becomes available dealing with more general weakly continuous maps, one may investigate the consequences for the Young measure.

9. Compactness with minimal separation of wave cone and manifold. In this section we shall prove Theorem 5.1 on compactness for the augmented system (1.9) of elasticity. We shall begin with several remarks dealing with entropy pairs and the Div-Curl Lemma in the context of a general system of conservation laws,

$$
\partial_{t} u+\partial_{x} f(u)=0, \quad u: R^{2} \rightarrow R^{p}
$$

in one space dimension. We note that if $(\eta, q)$ is an entropy pair for (9.1) then so is $\left(Q \eta, Q^{*} q\right)$ where

$$
\begin{gathered}
Q \eta=\eta(u)-\eta(\alpha)-\nabla \eta(\alpha)(u-\alpha), \\
Q^{*} q=q(u)-q(\alpha)-\nabla \eta(\alpha)\{f(u)-f(\alpha)\},
\end{gathered}
$$

and $\alpha$ is an arbitrary vector in $R^{p}$. The quadratic part $Q \eta$ of $\eta$ at $\alpha$ is obtained by subtracting the linear part $Q \eta=O(u-\alpha)^{2}$. As a consequence of the compatibility condition (4.6) it follows that $Q^{*} q=O(u-\alpha)^{2}$. Thus, if an entropy field

$$
\partial_{t} \eta\left(u^{\varepsilon}\right)+\partial_{x} q\left(u^{\varepsilon}\right)
$$

associated with a weakly convergent sequence of solutions $u^{\varepsilon}$ to (9.1) is compact in $W^{-1,2}$, then so is its quadratic part

$$
\partial_{t} Q \eta\left(u^{\varepsilon}, \alpha\right)+\partial_{x} Q^{*} q\left(u^{\varepsilon}, \alpha\right)
$$

for all $\alpha$. In particular, the Div-Curl Lemma implies that

$$
\left\langle\nu, Q \eta_{1} Q^{*} q_{2}-Q \eta_{2} Q^{*} q_{1}\right\rangle=\left\langle\nu, Q \eta_{1}\right\rangle\left\langle\nu, Q^{*} q_{2}\right\rangle-\left\langle\nu, Q \eta_{2}\right\rangle\left\langle\nu, Q^{*} q_{1}\right\rangle
$$

for all entropy pairs $\left(\eta_{j}, q_{j}\right)$ for which the corresponding entropy fields (9.4) are compact. 
We may rephrase this observation in the following way: If $\nu$ is a measure which commutes with $B$ acting on pairs $(\eta, q)$ with $\eta$ linear in $u$, i.e. if

$$
\left\langle\nu, u_{i} f_{k}-u_{k} f_{i}\right\rangle=\left\langle\nu, u_{i}\right\rangle\left\langle\nu, f_{k}\right\rangle-\left\langle\nu, u_{k}\right\rangle\left\langle\nu, f_{i}\right\rangle
$$

for $1 \leqslant i, k \leqslant p$, and if $\nu$ commutes with $B$ acting on two particular pairs $\left(\eta_{j}, q_{j}\right)$, i.e. if

$$
\left\langle\nu, \eta_{1} q_{2}-\eta_{2} q_{1}\right\rangle=\left\langle\nu, \eta_{1}\right\rangle\left\langle\nu, q_{2}\right\rangle-\left\langle\nu, \eta_{2}\right\rangle\left\langle\nu, q_{1}\right\rangle,
$$

then $\nu$ commutes with $B$ acting on the quadratic parts (9.2) and (9.3) of $\left(\eta_{j}, q_{j}\right)$, i.e. (9.5) holds.

In the setting of Lagrangian elasticity, we shall show that the associated Young measure is a Dirac mass by analyzing the locally fourth order commutativity relation (9.5) where $\left(\eta_{1}, q_{1}\right)$ is the pair associated with mechanical energy (1.10) and $\left(\eta_{2}, q_{2}\right)$ is the dual pair (1.11).

THEOREM 9.1. Let $g_{i j}$ denote the flux functions (5.5) of the augmented system (1.9) and suppose that $\sigma^{\prime}>0$ and $\sigma^{\prime \prime} \neq 0$. If $\nu$ is an arbitrary probability measure on $R^{2}$ such that

$$
\left\langle\nu, g_{i 1} g_{k 2}-g_{i 2} g_{k 1}\right\rangle=\left\langle\nu, g_{i 1}\right\rangle\left\langle\nu, g_{k 2}\right\rangle-\left\langle\nu, g_{i 2}\right\rangle\left\langle\nu, g_{k 1}\right\rangle
$$

for $1 \leqslant i, k \leqslant 4$, then $\nu$ is a Dirac mass if the diameter of its support is sufficiently small.

Proof. We shall regard (9.1) as consisting of two primitive conservation laws associated with mass and momentum

$$
\partial_{t} v-\partial_{x} u=\varphi_{1}, \quad \partial_{t} u-\partial_{x} \sigma(v)=\varphi_{2}
$$

together with two extensions

$$
\partial_{t} \eta_{j}+\partial_{x} q_{j}=\varphi_{j+2}, \quad j=1,2,
$$

given by (1.10) and (1.11). From the remarks above it follows that

$$
\left\langle\nu, Q g_{i 1} Q^{*} g_{k 2}-Q g_{i 2} Q_{k 1}^{*}\right\rangle=\left\langle\nu, Q g_{i 1}\right\rangle\left\langle\nu, Q^{*} g_{k 2}\right\rangle-\left\langle\nu, Q g_{i 2}\right\rangle\left\langle\nu, Q^{*} g_{k 1}\right\rangle
$$

for $1 \leqslant i, k \leqslant 4$. We note that if $i=3$ and $k=4$ then (9.7) becomes (9.5). We shall make use of (9.7) with $\alpha$ equal to the center of mass $(\bar{u}, \bar{v})$ of $\nu$. As a consequence of Galilean invariance, we may assume that $\bar{u}=0$.

A simple calculation shows that

$$
\begin{array}{ll}
Q \eta_{1}=\frac{1}{2} u^{2}+Q \Sigma, & Q^{*} q_{1}=-u \Delta \sigma, \\
Q \eta_{2}=u w, & Q^{*} q_{2}=-\left(\frac{1}{2} u^{2}+\bar{Q} \Sigma\right),
\end{array}
$$

where $Q \Sigma$ denotes the quadratic part of the primitive $\Sigma$ of $\sigma$,

$$
\begin{aligned}
Q \Sigma & \equiv Q^{\Sigma}(v, \bar{v})=\Sigma(v)-\Sigma(\bar{v})-\Sigma^{\prime}(\bar{v})(v-\bar{v}), \\
\bar{Q} \Sigma & \equiv Q^{\Sigma}(\bar{v}, v)=\Sigma(\bar{v})-\Sigma(v)-\Sigma^{\prime}(v)(\bar{v}-v), \\
w & =v-\bar{v}, \quad \text { and } \quad \Delta \sigma=\sigma(v)-\sigma(\bar{v}) .
\end{aligned}
$$


With the aid of (9.8) we may write (9.5) in the form $L=R$ where

$$
\begin{gathered}
L=\left\langle\nu, \frac{1}{4} u^{4}+Q \Sigma \bar{Q} \Sigma-\frac{1}{2} u^{2} \Delta \sigma w\right\rangle, \\
R=\left\langle\nu, \frac{1}{2} u^{2}+Q \Sigma\right\rangle\left\langle\nu, \frac{1}{2} u^{2}+\bar{Q} \Sigma\right\rangle-\langle\nu, u \Delta \sigma\rangle\langle\nu, u w\rangle .
\end{gathered}
$$

We have merely carried out the multiplication in equation (9.5) and used the fact that $Q \Sigma+\bar{Q} \Sigma=\Delta \sigma w$ since $\Sigma^{\prime}=\sigma$. We shall first analyze the fourth order terms in the integrand $I$ of $L$. We have

$$
I=\left\{\frac{1}{4} u^{4}+Q \Sigma \bar{Q} \Sigma-\frac{1}{2} \sigma^{\prime}(\bar{v}) u^{2} w^{2}\right\}-\frac{1}{2} u^{2} Q \sigma w
$$

since the increment $\Delta \sigma$ of $\sigma$ may be written in the form

(9.11) $\Delta \sigma=\sigma^{\prime}(\bar{v}) w+Q \sigma, \quad$ where $Q \sigma=\sigma(v)-\sigma(\bar{v})-\sigma^{\prime}(\bar{v})(v-\bar{v})$.

The locally fourth order part of $I$ is contained in brackets. The main step in simplifying the equation $L=R$ is to analyze the expected value of the mixed term, i.e.

$$
\left\langle\nu, \frac{1}{2} \sigma^{\prime}(\bar{v}) u^{2} w^{2}\right\rangle
$$

which appears in the fourth order part of $L$ in (9.9). A useful expression for (9.12) is given below in (9.21) and is obtained by pairing the forms associated with mechanical energy and momentum and the forms associated with dual energy and mass as follows: From (9.7) we obtain

$$
\left\langle\nu,-\Delta \sigma\left(\frac{1}{2} u^{2}+Q \Sigma\right)-u^{2} \Delta \sigma\right\rangle=-\langle\nu, Q \sigma\rangle\left\langle\nu, \frac{1}{2} u^{2}+Q \Sigma\right\rangle
$$

using $i=2$ (momentum) and $k=3$ (mechanical energy). Simplifying (9.13) yields an equation

$$
\left\langle\nu, \Delta \sigma\left(\frac{1}{2} u^{2}-Q \Sigma\right)\right\rangle=-\langle\nu, Q \sigma\rangle\left\langle\nu, \frac{1}{2} u^{2}+Q \Sigma\right\rangle
$$

from which we shall extract a useful expression for the quantity (9.12). First, we decompose the integrand on the left-hand side of (9.14) into its cubic and quadratic parts

$$
\Delta \sigma\left(\frac{1}{2} u^{2}-Q \Sigma\right)=\sigma^{\prime}(\bar{v}) w\left(\frac{1}{2} u^{2}-Q \Sigma\right)+Q \sigma\left(\frac{1}{2} u^{2}-Q \Sigma\right) .
$$

The cubic part of the expected value of (9.15) may be simplified by pairing mass and the dual energy, i.e. by using the equation

$$
\left\langle\nu,-u^{2} w+w\left(\frac{1}{2} u^{2}+\bar{Q} \Sigma\right)\right\rangle=0 .
$$

Equation (9.16) follows from (9.7) with $i=1$ (mass) and $k=4$ (dual energy). Here we have used the fact that the expected values of $w$ and $u$ vanish. Equation (9.16) simplifies to the statement that

$$
\left\langle\nu, w\left(\frac{1}{2} u^{2}-\bar{Q} \Sigma\right)\right\rangle=0
$$

Therefore

$$
\left\langle\nu, \sigma^{\prime}(\bar{v}) w\left(\frac{1}{2} u^{2}-\bar{Q} \Sigma\right)\right\rangle=0 .
$$

In order to take advantage of (9.17) we rewrite (9.15) in the form

$$
\begin{aligned}
\Delta \sigma\left(\frac{1}{2} u^{2}-Q \Sigma\right)= & \sigma^{\prime}(\bar{v}) w\left(\frac{1}{2} u^{2}-\bar{Q} \Sigma\right) \\
& +\sigma^{\prime}(\bar{v}) w(\bar{Q} \Sigma-Q \Sigma Q \Sigma)+Q \sigma\left(\frac{1}{2} u^{2}-Q \Sigma\right) .
\end{aligned}
$$


Using (9.17) and (9.18) we may reformulate (9.14) as follows:

$$
\left\langle\nu, \sigma^{\prime}(\bar{v}) w(\bar{Q} \Sigma-Q \Sigma)+Q \sigma\left(\frac{1}{2} u^{2}-Q \Sigma\right)\right\rangle=-\langle\nu, Q \sigma\rangle\left\langle\nu, \frac{1}{2} u^{2}+Q \Sigma\right\rangle,
$$

or more compactly as

$$
\left\langle\nu, \frac{1}{2} u^{2} Q \sigma-F\right\rangle=S,
$$

where

$$
S=-\langle\nu, Q \sigma\rangle\left\langle\nu, \frac{1}{2} u^{2}+Q \Sigma\right\rangle \quad \text { and } F=Q \sigma Q \Sigma-\sigma^{\prime}(\bar{v}) w(\bar{Q} \Sigma-Q \Sigma) .
$$

We note that locally fourth order form $F$ depends only on $v$ and $\bar{v}$. The mixed product $u^{2} Q \sigma$ appearing on the left of (9.19) is a multiple of the quantity $-\frac{1}{2} \sigma^{\prime}(\bar{v}) u^{2} w^{2}$ whose expected value we are trying to analyze, modulo higher order terms. To see this, we write

$$
Q \sigma=\frac{1}{2} \sigma^{\prime \prime}(\bar{v}) w^{2}+C \sigma
$$

where $C \sigma$ is the cubic part of $\sigma$ at $\bar{v}$, so that

$$
\frac{1}{2} u^{2} Q \sigma=\frac{1}{4} \sigma^{\prime \prime}(\bar{v}) u^{2} w^{2}+\frac{1}{2} u^{2} C \sigma .
$$

Substituting (9.20) into (9.19) yields

$$
\left\langle\nu, \frac{1}{4} \sigma^{\prime \prime}(\bar{v}) u^{2} w^{2}+\frac{1}{2} u^{2} C \sigma-F\right\rangle=S
$$

and consequently the desired result

$$
\begin{aligned}
\left\langle\nu,-\frac{1}{2} \sigma^{\prime} u^{2} w^{2}\right\rangle= & \left\langle\nu,-\left(2 \sigma^{\prime} / \sigma^{\prime \prime}\right) F+\left(\sigma^{\prime} / \sigma^{\prime \prime}\right) u^{2} C \sigma\right\rangle \\
& +\left\langle\nu,\left(2 \sigma^{\prime} / \sigma^{\prime \prime}\right) Q \sigma\right\rangle\left\langle\nu, \frac{1}{2} u^{2}+Q \Sigma\right\rangle,
\end{aligned}
$$

where $\sigma^{\prime}$ and $\sigma^{\prime \prime}$ are evaluated at $\bar{v}$.

Substituting (9.21) into the expected value of the integrand $I$ of $L$ allows us to rewrite the equation $L=R$ in the following form:

$$
\begin{aligned}
\left\langle\nu, \frac{1}{4} u^{4}+Q \Sigma \bar{Q} \Sigma-\left(2 \sigma^{\prime} / \sigma^{\prime \prime}\right) F+\left(\sigma^{\prime} / \sigma^{\prime \prime}\right) u^{2} C \sigma-\frac{1}{2} u^{2} Q \sigma w\right\rangle & \\
& +\left\langle\nu,\left(2 \sigma^{\prime} / \sigma^{\prime \prime}\right) Q \sigma\right\rangle\left\langle\nu, \frac{1}{2} u^{2}+Q \Sigma\right\rangle \\
= & \left\langle\nu, \frac{1}{4} u^{4}\right\rangle+\langle\nu, Q \Sigma\rangle\langle\nu, \bar{Q} \Sigma\rangle \\
& +\left\langle\nu, \frac{1}{2} u^{2}\right\rangle\langle\nu, Q \Sigma+\bar{Q} \Sigma\rangle-\langle\nu, u \Delta \sigma\rangle\langle\nu, u w\rangle .
\end{aligned}
$$

We shall show that a Taylor expansion around the center of mass of $\nu$ leads to an expression of the form

$$
\left\langle\nu, \frac{1}{4} u^{4}+\frac{1}{12} \tau w^{4}\right\rangle+\beta\langle\nu, u w\rangle^{2}+\frac{1}{4} \beta^{2}\left\langle\nu, w^{2}\right\rangle^{2}+H=\frac{1}{4}\left\langle\nu, u^{2}\right\rangle^{2}
$$

where $\beta=\sigma^{\prime}(\bar{v})$ and $H$ is a fifth order term with the structure

$$
\begin{aligned}
H= & \left\langle\nu, O\left(u w^{2}\right)\right\rangle\langle\nu, u w\rangle+\left\langle\nu, u^{2}\right\rangle\left\langle\nu, O\left(w^{3}\right)\right\rangle \\
& +\left\langle\nu, w^{2}\right\rangle\left\langle\nu, O\left(w^{3}\right)\right\rangle+\left\langle\nu, O\left(u^{2} w^{3}\right)+O\left(w^{5}\right)\right\rangle .
\end{aligned}
$$

Then we shall show that

$$
H \leqslant \text { const. } \sup |w|\left\{\left\langle\nu, w^{4}\right\rangle\right\}
$$


where the supremum of $w$ is taken over the support of $\nu$. Thus $H$ may be absorbed by the expected value of $\frac{1}{12} \beta^{2} w^{4}$ if the diameter of the support of $\nu$ is sufficiently small, producing an estimate of the form

$$
\left\langle\nu, \frac{1}{4} u^{4}+\frac{1}{24} \beta^{2} w^{4}\right\rangle \leqslant \frac{1}{4}\left\langle\nu, u^{2}\right\rangle^{2}
$$

since $\beta$ is positive. It follows from (9.25) and Jensen's inequality that $\nu$ is concentrated at $(\bar{u}, \bar{v})$.

In order to derive (9.23) from (9.22) we write (9.22) in the form

$$
\left\langle\nu, I_{1}\right\rangle+\sum_{j=1}^{3} T_{j}=\frac{1}{4}\left\langle\nu, u^{2}\right\rangle^{2}
$$

where

$$
\begin{aligned}
& T_{1}=\langle\nu, u \Delta \sigma\rangle\langle\nu, u w\rangle, \\
& T_{2}=\left\langle\nu, \frac{1}{2} u^{2}\right\rangle\left\langle\nu,\left(2 \sigma^{\prime} / \sigma^{\prime \prime}\right) Q \sigma-(Q \Sigma+\bar{Q} \Sigma)\right\rangle, \\
& T_{3}=\langle\nu, Q \Sigma\rangle\left\langle\nu,\left(2 \sigma^{\prime} / \sigma^{\prime \prime}\right) Q \sigma-\bar{Q} \Sigma\right\rangle, \\
& I_{1}=\frac{1}{2} u^{4}+Q \Sigma \bar{Q} \Sigma-\left(2 \sigma^{\prime} / \sigma^{\prime \prime}\right) F+\left(\sigma^{\prime} / \sigma^{\prime \prime}\right) u^{2} C \sigma-\frac{1}{2} u^{2} Q \sigma w .
\end{aligned}
$$

We shall decompose each of the terms $T_{j}$ into their fourth and fifth order parts. The collection of fifth order parts constitutes $H$. To begin, we write $T_{1}$ in the form

$$
T_{1}=\beta\langle\nu, u w\rangle^{2}+\langle\nu, u Q \sigma\rangle\langle\nu, u w\rangle,
$$

using (9.11). The second term of $T_{1}$ in (9.27) is fifth order and constitutes the first term of $H$ in (9.24). The term $T_{2}$ is itself fifth order and constitutes the second term of $H$ in (9.24). Indeed, the integrand in the second factor of $T_{2}$ may be written as $2\left(\sigma^{\prime} / \sigma^{\prime \prime}\right) Q \sigma-\Delta \sigma w$. Using $(9.11)$ we see that

$$
\Delta \sigma w=\sigma^{\prime} w^{2}+w Q \sigma, \quad 2\left(\sigma^{\prime} / \sigma^{\prime \prime}\right) Q \sigma=\sigma^{\prime} w^{2}+2\left(\sigma^{\prime} / \sigma^{\prime \prime}\right) C \sigma
$$

and hence that

$$
2\left(\sigma^{\prime} / \sigma^{\prime \prime}\right) Q \sigma-\Delta \sigma w=2\left(\sigma^{\prime} / \sigma^{\prime \prime}\right) C \sigma-w Q \sigma=O\left(w^{3}\right) .
$$

The term $T_{3}$ may be written in the form

$$
T_{3}=\langle\nu, Q \Sigma\rangle\left\langle\nu, \frac{1}{2} \sigma^{\prime} w^{2}\right\rangle+\langle\nu, Q \Sigma\rangle\left\langle\nu, 2\left(\sigma^{\prime} / \sigma^{\prime \prime}\right) C \sigma-\bar{C} \Sigma\right\rangle,
$$

the second part of which is fifth order, i.e.

$$
\langle\nu, Q \Sigma\rangle\left\langle\nu, 2\left(\sigma^{\prime} / \sigma^{\prime \prime}\right) C \sigma-\bar{C} \Sigma\right\rangle=\left\langle\nu, O\left(w^{2}\right)\right\rangle\left\langle\nu, O\left(w^{3}\right)\right\rangle
$$

and contributes to the third term of $H$ in (9.24). The expression (9.28) may be obtained by using the fact that

$$
\bar{Q} \Sigma=\frac{1}{2} \Sigma^{\prime \prime}(v)(\bar{v}-v)^{2}+C \bar{\Sigma}=\frac{1}{2} \sigma^{\prime}(v)(\bar{v}-v)^{2}+\bar{C} \Sigma
$$

and writing the integrand in the second factor of $T_{3}$ in the form

$$
\begin{aligned}
2 \frac{\sigma^{\prime}}{\sigma^{\prime \prime}} Q \sigma-\bar{Q} \Sigma & =\sigma^{\prime} w^{2}+2 \frac{\sigma^{\prime}}{\sigma^{\prime \prime}} C \sigma-\frac{1}{2} \sigma^{\prime} w^{2}-\bar{C} \Sigma \\
& =\frac{1}{2} \sigma^{\prime} 2^{2}+2 \frac{\sigma^{\prime}}{\sigma^{\prime \prime}} C \sigma-\bar{C} \Sigma .
\end{aligned}
$$


We conclude that (9.26) may be written as

$$
\left\langle\nu, I_{1}\right\rangle+\sigma^{\prime}\left\langle\nu, u w^{2}\right\rangle+\langle\nu, Q \Sigma\rangle\left\langle\nu, \frac{1}{2} \sigma^{\prime} w^{2}\right\rangle+H_{3}=\frac{1}{4}\left\langle\nu, u^{2}\right\rangle^{2}
$$

where $\mathrm{H}_{3}$ has the form of the first three terms of $H$. It remains to analyze the integrand $I_{1}$. To this end a Taylor expansion shows that

$$
\begin{aligned}
& F=\frac{1}{12} \sigma^{\prime} \sigma^{\prime \prime} w^{4}+O\left(w^{5}\right), \quad Q \Sigma \bar{Q} \Sigma=\frac{1}{4} \beta^{2} w^{4}+O\left(w^{5}\right), \quad \text { and } \\
& I_{1}=\frac{1}{4} u^{4}+\frac{1}{12} \beta^{2} w^{4}+O\left(w^{5}\right)+O\left(u^{2} w^{3}\right) .
\end{aligned}
$$

Thus the expected value of $I_{1}$ takes the form

$$
\left\langle\nu, I_{1}\right\rangle=\left\langle\nu, \frac{1}{4} u^{4}+\frac{1}{12} \beta^{2} w^{4}\right\rangle+\left\langle\nu, O\left(w^{5}\right)+O\left(u^{2} w^{3}\right)\right\rangle .
$$

The second term on the right-hand side of (9.30) constitutes the fourth term of $H$. We finally remark that the third term on the left side of (9.29) may be written as

$$
\langle\nu, Q \Sigma\rangle\left\langle\nu, \frac{1}{2} \sigma^{\prime} w^{2}\right\rangle=\frac{1}{4} \beta^{2}\left\langle\nu, w^{2} u\right\rangle^{2}+\left\langle\nu, w^{2}\right\rangle\left\langle\nu, O\left(w^{3}\right)\right\rangle .
$$

The second term on the right side of (9.30) contributes to the third term of $H$. The proof of (9.23) is complete.

The proof of Theorem 9.1 will be complete once we verify (9.24a). The proof of (9.24a) makes use of the observation that

$$
\left\langle\nu, u^{2}\right\rangle \leqslant \text { const. }\left\langle\nu, w^{2}\right\rangle
$$

which follows immediately from (9.6) with $i=1$ and $k=2$. Using (9.32) and Holder's inequality we see that the first three terms of $H$ are dominated by $\sup |w|\left\langle\nu, w^{2}\right\rangle^{2}$. Using (9.21) we may handle the fourth term of $H$ in a similar way.

10. Separation of wave cone and manifold for transonic flow. The purpose of this section is to record the observation that the small disturbance equations augmented by either of the two entropy forms associated with spatial translation invariance has the property that the wave cone is separated from the constitutive manifold. The small disturbance equations of steady two-dimension flow have exactly the same form as Lagrangian elasticity (5.1) where $t$ represents a spatial coordinate and $\sigma(v)=c v^{2}$ where $c$ is a positive constant. Thus the small disturbance equations are hyperbolic if $v>0$ and elliptic if $v<0$.

The aforementioned separation property is equivalent to the following statement: If the function

$$
(u, v)=\left\{\begin{array}{ll}
\left(u_{1}, v_{1}\right) & \text { for } y \cdot s>0 \\
\left(u_{2}, v_{2}\right) & \text { for } y \cdot s<0
\end{array}\right\}
$$

is a two-state distributional solution of either of the homogeneous systems

$$
\sum_{j=0}^{1} \partial_{j} g_{i j}(u, v)=0
$$

with $i=1,2,3$ or with $i=1,2,4$, then $u_{1}=u_{2}$ and $v_{1}=v_{2}$. Here the flux functions $g_{i j}$ are given by (5.5), and $s=\left(s_{0}, s_{1}\right)$ denotes the normal to the line of discontinuity which separates the constant state $\left(u_{1}, v_{1}\right)$ from the constant state $\left(u_{2}, v_{2}\right)$ in the 
$y_{1}-y_{2}$ plane. In other words, there exist no nontrivial discontinuities that simultaneously conserve mass momentum and either of the two dual entropy field. The proof is based upon a simple calculation using the following list of Rankine-Hugoniot relations:

$$
\begin{gathered}
s_{0}[v]-s_{1}[u]=0, \\
s_{0}[u]-s_{1}[\sigma]=0, \\
s_{0}\left[\frac{1}{2} u^{2}+\Sigma\right]-s_{1}[u \sigma]=0, \\
s_{0}[u v]-s_{1}\left[\frac{1}{2} u^{2}+\tau\right]=0,
\end{gathered}
$$

where $[v]=v_{1}-v_{2}$, etc. and $\tau$ is the Legendre transform of $\Sigma$. If (10.1) solves (10.2) with $i=1,2,3$ then (10.3)-(10.5) are satisfied. If (10.1) satisfies (10.2) with $i=1,2,4$ then (10.3), (10.4) and (10.6) are satisfied. We claim that if either of these last two statements hold then $\left(u_{1}, v_{1}\right)$ and $\left(u_{2}, v_{2}\right)$ coincide. This fact may be established as follows. Equations (10.3) and (10.5) imply that

$$
[v][u \sigma]-[u]\left[\frac{1}{2} u^{2}+\Sigma\right]=0
$$

since $s$ is nonzero. Without loss of generality we may assume that

$$
\bar{u}=\frac{1}{2}\left(u_{1}+u_{2}\right)=0 \text {. }
$$

Using (10.8) and the fact that $[a b]=\bar{a}[b]+\bar{b}[a]$ we may rewrite (10.8) in the form $[v]\left(2 u_{1} \bar{\sigma}\right)-2 u_{1}[\Sigma]=0$, or equivalently

$$
\bar{\sigma}[v]=[\Sigma]
$$

In a similar way, equations (10.4) and (10.6) imply that

$$
\bar{v}[\sigma]=[\tau] \text {. }
$$

We notice that (10.9) and (10.10) are equivalent since $\tau=v \sigma(v)-\Sigma(v)$. In particular, any discontinuity conserving mass and momentum has the property that it conserves the entropy field associated with $\left(\eta_{1}, q_{1}\right)$ defined by (1.10) if and only if it conserves the dual entropy field associated with $\left(\eta_{2}, q_{2}\right)$ defined by (1.11). Thus, the separation property of cone and manifold for the aforementioned augmented systems of three equations simultaneously holds or fails.

We observe that (10.9) and (10.10) may be written in the form

$$
L \equiv \frac{1}{2}\left\{\sigma\left(v_{1}\right)+\sigma\left(v_{2}\right)\right\}=\left\{\Sigma\left(v_{1}\right)-\Sigma\left(v_{2}\right)\right\}\left(v_{1}-v_{2}\right) \equiv R .
$$

We note that if $\sigma$ is convex then $L \leqslant R$. Furthermore, equality holds for a strictly convex function $\sigma$ if and only if $v_{1}=v_{2}$. Thus the separation property holds for the systems (10.2) if $\sigma$ is strictly convex.

We remark that the doubly augmented system (1.9) also has the separation property if $\sigma$ is strictly convex. It would be interesting to try to extend the analysis of $\S 9$ to the case where $\sigma^{\prime}$ changes sign.

\section{REFERENCES}

1. J. M. Ball, On the calculus of variations and sequentially weakly continuous maps, Lecture Notes in Math. vol. 564, Springer-Verlag, 1976.

2. Convexity conditions and existence theorems in nonlinear elasticity, Arch. Rational Mech. Anal. 63 (1977), 337-403.

3. J. M. Ball, J. C. Currie and P. J. Olver, Null Lagrangians, weak continuity and variational problems of arbitrary order, J. Funct. Anal. 41 (1981), 135-175. 
4. B. Dacorogna, Weak continuity and weak lower semicontinuity of nonlinear functionals, Lecture Notes in Math., Springer-Verlag, 1982.

5. R. J. DiPerna, Uniqueness of solutions to hyperbolic conservation laws, Indiana Univ. Math. J. 28 (1979), 137-188.

6. Convergence of approximate solutions to conservation laws, Arch. Rational Mech. Anal. 82 (1983), 27-70.

7. Convergence of the viscosity method for isentropic gas dynamics, Comm. Math. Phys. 91 (1983), 1-30.

8. Measure-valued solutions to conservation laws, Arch. Rational Mech. Anal. 88 (1985), 223-270

9. J. Glimm and P. D. Lax, Decay of solutions of systems of nonlinear hyperbolic conservation laws, Amer. Math. Soc. Memoir 101 (1970).

10. B. Keyfitz, Solutions with shocks, an example of an $L^{1}$-contractive semigroup, Comm. Pure Appl. Math. 24 (1971), 125-132.

11. P. D. Lax, Hyperbolic systems of conservation laws. II, Comm. Pure Appl. Math. 10 (1957), 537-566.

12. Shock waves and entropy, Contributions to Nonlinear Functional Analysis (E. A Zarantonello, ed.), Academic Press, 1971.

13. F. Murat, Compacité par compensation, Ann. Scuola Norm. Sup. Pisa 5 (1978), 489-507.

14. Compacité par compensation: Condition necessaire et suffisante de continuité faible sous une hypotheses de rang constant, Ann. Scuola Norm. Sup. Pisa 8 (1981), 69-102.

15. L. Tartar, Compensated compactness and applications to partial differential equations, Research Notes in Math., Nonlinear Analysis and Mechanics: Heriot-Watt Symposium, Vol. 4 (R. J. Knops, ed.), Pitman Press, 1979.

16. The compensated compactness method applied to systems of conservation laws, Systems of Nonlinear Partial Differential Equations (J. M. Ball, ed), NATO ASI Series, C. Reidel, 1983.

17. Solutions oscillantes des equations de Carleman, Séminaire Goulaouic-Meyer-Schwarz, January 1983.

18. A. I. Vol' pert, The spaces BV and quasilinear equations, Math. USSR-Sb. 2 (1967), 257-267.

19. D. Serre, private communication.

20. N. Kruzkov, First order quasilinear equations in several independent variables, Math. USSR-Sb. 10 (1970), 127-243.

21. C. B. Morrey, Jr., Multiple integrals in the calculus of variations, Springer, Berlin, 1966.

Department of Mathematics, Duke University, Durham, North Carolina 27706

Current address: Department of Mathematics, University of California, Berkeley, California 94720 